This is the accepted manuscript of the article:

"New features for delamination depth evaluation in carbon fiber reinforced plastic materials using eddy current pulse-compression thermography",

Authors: Q. Yi, G.Y. Tian, H. Malekmohammadi, J. Zhu, S. Laureti, M. Ricci, published in NDT \& E International, Volume 102, 2019, Pages 264-273

ISSN 0963-8695, https://doi.org/10.1016/j.ndteint.2018.12.010

(http://www.sciencedirect.com/science/article/pii/S0963869518305826)

(c) (1) $\circledast()$

The present manuscript is an open access article distributed under the terms and conditions of the Creative Commons Attribution (CC BY-NC-ND)

BY NC ND license (http://creativecommons.org/licenses/by/4.0/) 


\title{
New features for delamination depth evaluation in carbon fiber reinforced plastic materials using eddy current pulse-compression thermography
}

\author{
Q. Yi ${ }^{1}$, G.Y. Tian ${ }^{1}$, H. Malekmohammadi ${ }^{2}$, J. Zhu' ${ }^{1}$, S. Laureti ${ }^{2}$, M. Ricci ${ }^{3}$ \\ ${ }^{I}$ Department of Electrical and Electronic Engineering, Newcastle University, Merz Court, NE1 7RU, Newcastle \\ upon Tyne, UK \\ ${ }^{2}$ Department of Engineering, University of Perugia, Polo Scientifico didattico di Terni, Strada di Pentima 4, \\ 05100 Terni, ITALY \\ ${ }^{3}$ Department of Informatics, Modeling, Electronics and System Engineering, University of Calabria, \\ Rende, 87036, ITALY
}

\begin{abstract}
The growing application of composite materials in aerospace leads to the urgent need of non-destructive testing and evaluation (NDT\&E) techniques capable of detecting defects such as impact damage and delamination possibly existing in those materials. Eddy current pulsed thermography is an emerging non-destructive testing (NDT) technique capable of detecting such defects. However, characterization of delamination within composite materials is difficult to be achieved by a single pulse excitation, especially in carbon fiber reinforced plastic materials as the extraction of thermal diffusion in such multi-layered structures is challenging. To cope with this problem of signal-to-noise ratio, this paper proposes the eddy current pulse-compression thermography $(\mathrm{ECPuCT})$, combining the Barker code modulated eddy current excitation and pulse-compression technique to enhance the capability of characterizing delamination on carbon fiber reinforced plastic materials. Additionally, a thermal pattern enhanced method based on kernel principal component analysis technique is used to locate the delaminated areas. Two features, including a newly proposed crossing point of impulse responses related to defective and non-defective areas and skewness of impulse responses are investigated for delamination depth evaluation. Results show that delamination can be detected within depths ranging from $0.46 \mathrm{~mm}$ to $2.30 \mathrm{~mm}$ and both the proposed features have a monotonic relationship with delamination depths.
\end{abstract}

Keywords: Pulse-Compression; Eddy Current Pulsed Thermography; Feature extraction; Carbon Fiber Reinforced Plastic; Delamination depth; Barker code

\section{Introduction}

The use of Carbon Fiber Reinforced Plastic (CFRP) materials has become increasingly popular among conventional engineered materials due to their extraordinary mechanical and thermal properties, such as high strength-to-weight ratio, corrosion resistance, improved fatigue performance and low coefficient of thermal expansion [1]. Delamination is one of the most common defects for composite materials, which might arise either during the manufacturing process or during their in-service life, e.g. during the standard lifetime of aircraft components. A typical delamination initiates and grows between different plies of the composite, which may result in lowered in-plane strength and stiffness, eventually leading to catastrophic failure of the whole structure [2].

To detect delamination and ensure the safe operation of composite components, NDT techniques are used during both manufacturing and operation of the composite components. As an example, Ultrasonic Testing (UT) can locate the substantial flaws in CFRP via ultrasonic waves with frequencies usually ranging from hundreds of $\mathrm{kHz}$ to several tens of $\mathrm{MHz}$ [3].

However, ultrasonic wave can be attenuated either by the geometry and anisotropy of the material or via viscoelastic absorption loss in resin and multiple scattering loss caused by fibers. 
As a consequence, delamination echoes are possibly buried in noise, thus computationallyexpensive post-processing algorithms are often required to implement fruitfully the feature extraction [4]. X-Ray Computed Tomography (XCT) can provide both qualitative and quantitative information for a comprehensive understanding of delamination initiation and evolution, but the cost in terms of experimental setup (software and hardware) and the complexity of measurements makes such test quite challenging to be performed routinely [4].

A promising NDT technique to detect delamination in CFRP is the Active Thermography (AT) $[5,6]$. Being a non-contact method with the capability of inspecting a large area of the Sample Under Test (SUT), AT has been extensively used for inspecting CFRP in a number of different application scenarios [7], as well as for material characterization [8,9], cultural heritage diagnostic [10], on-line monitoring of goods in different production plants [11, 12]. In $\mathrm{AT}$, the desired thermal contrast is achieved by applying an external heating stimulus over the SUT [13] by means of various different physical sources. Light sources are the most common, see for instance [14-19], wherein the SUT's illuminated surface heats up and then the heat diffuses toward the inner side of the sample to restore thermal equilibrium [20]. Alternatively, other methods of excitation have been proposed and successfully applied such as Ultrasound Vibro-Thermography [21], Eddy-Current Pulsed Thermography (ECPT) [22] and Microwave Thermography [23]. Among these, one of the extensively applied AT technique is ECPT. ECPT uses a coil driven by alternating current to generate EC inside the SUT that increases temperature due to the Joule effect. Since CFRP exhibits low electrical conductivity, in most of the ECPT cases the stimulation can be considered volumetric since the typical eddy-current skin depths is greater than or comparable with the CFRP sample thickness [24]. For this reason, ECPT is also poorly influenced by the surface conditions of the SUT [18]. Despite the progresses made on ECPT, both for evaluating the so-called barely invisible impact damage [24-26] and visualizing delamination [27-29], the quantitative evaluation of the delamination depth is still challenging. In fact, the complex structure of CFRP, i.e. layered structure, anisotropic conductivity and different fiber direction, results in non-uniform thermal distribution, thus hampering the faithful quantitative evaluation of the delamination depth from the thermal response.

To gain insight about the here-proposed approach, it is worth briefly resuming here the possible ways in which AT can be performed. Despite the employed heating source, the classification of the AT schemes is strictly related to the time-frequency characteristic of the heating modulation signal. In Pulsed Thermography (PT), a short time duration heating stimulus - usually of few milliseconds- excites the SUT within an extended bandwidth. The duration of the pulse is significantly shorter than typical heat diffusion phenomena in the SUT, so that the excitation pulse well-approximates the Dirac's delta $\delta(t)$. Features extraction can be performed by analysing both the heating and the cooling trends of the recorded impulse response $h(t)$ pixelwise. Note that the maximum achievable value of the Signal-to-Noise Ratio (SNR) in PT is set by the employed excitation source power. Improved SNR values might be obtained by concentrating the heating stimulus energy within a narrow frequency range as in the Lock-in Thermography (LT) scheme, wherein the heating stimulus is modulated by a sinusoid at given frequency. The main drawback of LT is the less amount of possible information obtainable with respect to PT [30], which is a direct consequence of the narrower bandwidth of the heating stimulus. Furthermore, if the delamination depths are aimed at being characterized by LT, an improper choice of the modulation frequency might lead to thermal diffusion length values that do not match properly the possible defects depths [24].

In the last decades, efforts have been made to merge the effectiveness of PT and the SNR values achievable via LT, leading for examples to Pulsed Phase Thermography and MultiFrequency Lock-in Thermography [31, 32]. An alternative approach exploits the positive features of Pulse-compression (PuC) technique and coded excitations. The use of coded excitation signals and $\mathrm{PuC}$ in thermal phenomena has been pioneered by research groups led by Mandelis [33] and Mulaveesala [34]. Subsequently, various Pulse-Compression Thermography (PuCT) procedures have been proposed $[35,36]$. The $\mathrm{PuC}$ algorithm outputs a good estimate of impulse response that is close in both characteristics and quality to that obtainable from PT. In $\mathrm{PuCT}$, the heat source emission is commonly modulated either by a frequency-modulated 
"Chirp" signal [37], or by a phase-modulated signal [34], e.g. Barker code. These coded excitations have a characteristic trait: their bandwidth $B$ and their time duration $T$ are uncorrelated. This means that the frequency content of the coded signal can be tailored to suit the investigation of a given sample, while $T$ can be increased almost arbitrarily to achieve the desired SNR [38, 39]. In addition, both time and frequency domain analyses can be performed for characterizing the investigated material when coded excitations are used. In particular, the time analysis can be retrieved after the application of the $\mathrm{PuC}$ algorithm, whilst a frequency analysis can be directly implemented on the raw acquired data. Although PuCT is now a quite established technique that shows promises for maximizing the SNR even when low power heat sources are employed, very few attempts are reported in literature to extend this approach to ECPT $[40,41]$.

Based on the above-mentioned PuC features, the aims of this work are (i) to increase the detectability of delamination and (ii) to evaluate them quantitatively on an artificiallydelaminated CFRP sample by using coded waveforms and PuC technique in ECPT. Hereinafter, such a combination will be referred as Eddy Current Pulse-Compression Thermography (ECPuCT).

An inverse method calculates parameters from a set of experimental data for evaluation of defects, while a forward method acts in the opposite way. Currently, inverse evaluation techniques for thermography can be divided into two categories: feature-based and modelbased methods. The features for ECPT systems are represented by thermal distribution in thermal images and transient responses in time domain. The PCA [25, 42, 43], ICA [25], Fourier transform [44] and wavelet transform [45] techniques have been applied for defect detection and identification in time, frequency or spatial domains. Model-based inverse techniques, for example, Virtual Wave [46] and FEM (finite element modelling) [47] can lead to multiple parameters analysis and are able to reconstruct the profile of the defects. But regression algorithms and regularization processes [48] are needed in model-based inverse techniques due to ill-posed problems, they are more computation-expensive and less accurate. The Kernel Principal Component Analysis (K-PCA) used in this work as a feature-based inverse technique is conducted in time domain, which is computation-inexpensive and can provide clear relationship between defects and features, proving the possibility of real-time and online inspection in the future. It has been chosen to adopt K-PCA technique for detecting the defective area, and to evaluate the delamination depth with proposed simple cross-point feature from impulse responses.

Fig. 1 shows the block diagram of the overall process to extract features for the delamination depths characterization using ECPuCT. Each block of this diagram, numbered from 1 to 4 , shows the steps followed in this study to extract the final feature. ECPuCT method is firstly applied on the CFRP benchmark sample containing manmade delamination defects at different depths and the raw data, in the form of thermograms, is acquired, as for block 1 . Then a denoising algorithm is applied on each individual pixel's signal to remove noise, and a nonlinear fitting function is also exploited to remove the step-heating contribution from the denoised data -as explained in [19]- see Fig.1 block 2. Then, as showed in block 3, the calculation of the impulse response $h(t)$ is performed by convolving the de-trended signal with a matched filter. After obtaining the impulse response $h(t)$ of each individual pixel, the feature extraction process is carried out, as depicted in block 4. K-PCA technique is used on the retrieved $h(t)$ 's to obtain principal components and to enhance delaminated areas. Based on the enhanced delaminated area, the impulse responses including delaminated and nondelaminated areas are selected manually. Different features including the proposed crossing point of $h(t)$ 's and skewness of defective areas impulse responses are validated and compared for delamination depth evaluation. 
This paper is organized as follows: Section 2 presents the theoretical background of EC stimulated heating, the basic theory of $\mathrm{PuC}$, the proposed methodology of impulse response based thermal enhanced pattern technique and the feature extraction process. Section 3 provides details about the Barker code excitation, the ECPuCT experimental setup and the benchmark delaminated CFRP sample. Section 4 shows how to perform PuC correctly and highlights the analysis of various extracted thermal pattern by the proposed technique. The validation of the proposed feature is also conducted through experiment. Conclusion will be made in Section 5.

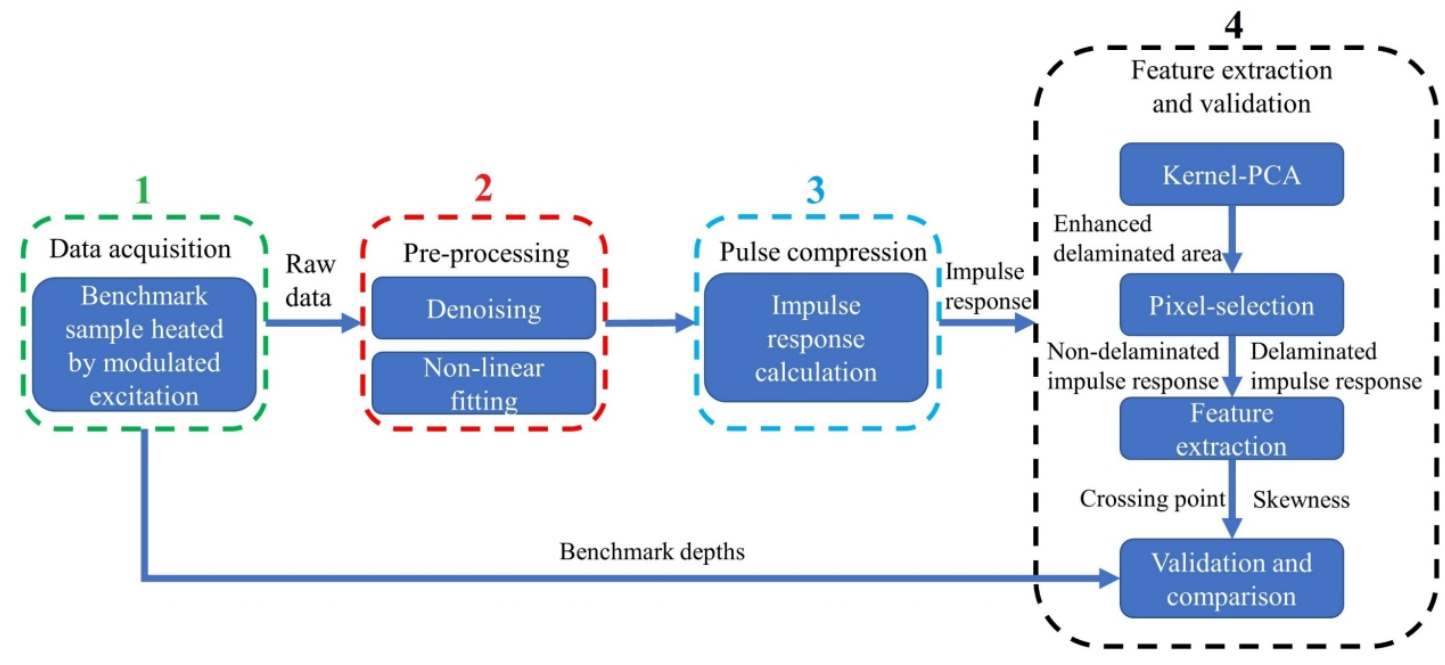

Fig. 1 Block diagram of the processing steps of proposed feature extraction and validation

\section{Theoretical background of eddy current pulse compression thermography and proposed feature extraction}

\subsection{Theory of eddy current stimulated volumetric heating in CFRP}

The theory of ECPT on ferromagnetic material has been addressed in previous researches [49-51]. The principle of ECPT for CFRP is different from that of ferromagnetic materials, concerning both the heating mode and the thermal wave diffusion pattern. According to Eq.(1), the penetration depth $\delta$ of an electromagnetic wave into a conductive material has a monotonic decreasing relationship with increasing current frequency:

$$
\delta=\frac{1}{\sqrt{\pi \mu \sigma f_{\text {carrier }}}}
$$

where $f_{\text {carrier }}$ is the frequency of the excitation current $[\mathrm{Hz}], \sigma$ is electrical conductivity $\left[\mathrm{S} \cdot \mathrm{m}^{-1}\right]$, and $\mu$ is magnetic permeability [H.m $\mathrm{m}^{-1}$. In general, for CFRP the bulk conductivity $\sigma$ value is in the order of $15000 \mathrm{~S} \cdot \mathrm{m}^{-1}$ and it is non-magnetic [52]. Considering an $f_{\text {carrier }}=300 \mathrm{kHz}$, the corresponding penetration depth is $7.50 \mathrm{~mm}$ while for the same value of $f_{\text {carrier }}$, the penetration depth in steel $\left(\sigma=9.93 \times 10^{6} \mathrm{~S} / \mathrm{m}, \mu=3.77 \times 10^{-4} \mathrm{H} / \mathrm{m}\right)$ is equal to $29.08 \mu \mathrm{m}$, which is significantly smaller than one obtained for CFRP. Thus, since the thickness of the CFRP sample is often lower than corresponding value of $\delta$, the heating mode is volumetric.

Due to skin effect, the eddy current density has an exponential decay in through-thickness direction. Therefore, the heat generated through Joule effect at different layer of composites decays exponentially through the perpendicular direction.

The thermal response in CFRP can be recognized as a sum of thermal waves. Each of them has a different frequency $f$, thermal diffusion length $\mu$, and diffusion velocity $v$. According to thermal wave theory, $\mu$ can be expressed as:

$$
\mu=\sqrt{\frac{k}{\pi f \rho C}}=\sqrt{\frac{\alpha}{\pi f}}
$$


where $k$ is thermal conductivity, $\rho$ is density $\left[g \cdot m^{-3}\right], C$ is heat capacity $\left[J \cdot g^{-1} \cdot K^{-1}\right.$ ], and $\alpha$ is thermal diffusivity $\left[W \cdot m^{-1} \cdot K^{-1}\right]$. The diffusion velocity $v_{p}$ can be obtained as:

$$
v_{p}=2 \sqrt{\pi f \alpha}
$$

Equations (2) and (3) indicate that thermal waves having higher frequencies diffuse faster but shallower, while low frequency thermal waves diffuse slower but deeper into the sample. If there is a defect at some depth, the parameters (like amplitude and phase) of the thermal wave will change, thus allowing the flaws to be detected by an InfraRed (IR) camera.

In the case of volumetric heating, the ECPT characterization can be either in transmission or reflection mode. The transmission and reflection arrangements are depicted in Fig.2 (a). If the thermal diffusion length $\mu$ is greater than depth $d$ at which the delamination/defect is possibly buried, response from the defect can be observed through the acquired surface temperature field. The unique advantage of the volumetric heating compared to the surface heating scenarios (e.g. as in the case of light-stimulated thermography) comes from the direct interaction between the buried defect and the induced eddy current field, see Fig.2 (b). In surface heating mode, the generated thermal wave must be reflected by the defect, taking twice the time with respect to the volumetric heating mode before being recorded by the IR camera. Overall, the heating pattern of the EC excitation in transmission arrangement can be considered as follows: firstly, the whole specimen is heated-up by the induced eddy current and Joule's effect [26]. Then, the defected area is heated more than the sound areas at the same depth. The information needed for characterizing the defects comes from the thermal wave travelling from the defected area at a depth $d$, as shown in Fig.2 (a), where $t_{r}$ and $t_{d}$ represent the travelling time of the thermal response from defect to the surface in transmission and reflection modes respectively.

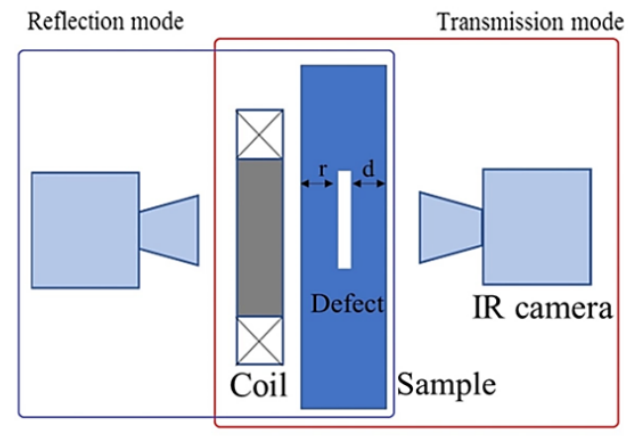

(a)

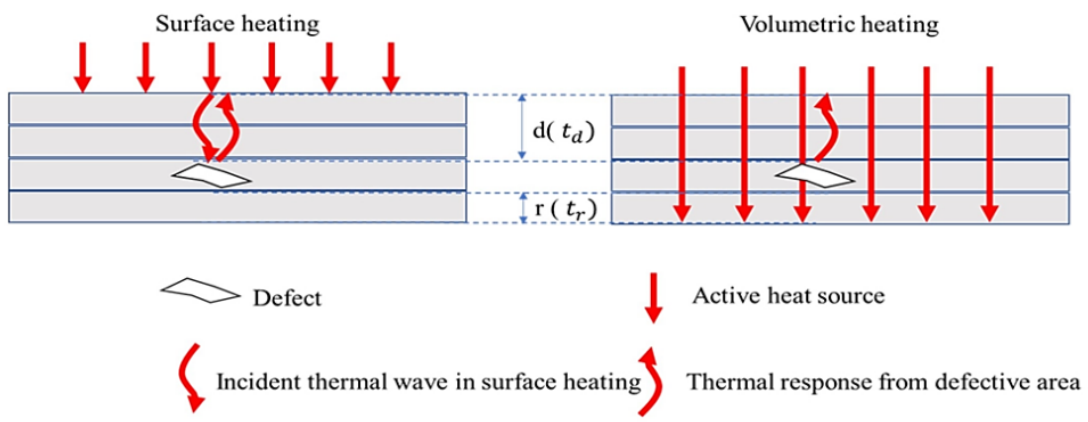

(b)

Fig. 2 Eddy current pulsed thermography measurement modes, (a) Configuration of transmission and reflection modes, (b) Comparison of surface and volumetric heating and their effect on the detection time. 


\subsection{Pulse compression basic theory}

This section will give a detailed explanation of the $\mathrm{PuC}$ technique. $\mathrm{PuC}$ is a wide-spread measurement technique used to experimentally estimate the impulse response of a Linear Time Invariant (LTI) system in a noisy environment or in presence of very low SNR values [53]. In standard PT, flash lamps are commonly employed to excite the sample -assumed as an LTI system- within a time significantly shorter than the typical cooling time of the sample itself. Therefore, the so-provided heating stimulus can be modelled as a Dirac's Delta function $\delta(t)$, and the corresponding output $y(t)$, i.e. the pixel temperature/emissivity amplitude recorded with the elapsing time, is a good approximation of the impulse response $h(t)$ [19]. Features of interest are obtained by analyzing the $h(t)$ within a chosen range of interest $T_{h}$ as showed in Fig.3 (a). As it is also shown in Fig.3 (a), in PT the excitation is considered instantaneous and the sample impulse thermal response is measured for a time of interest $T_{h}$, which is the impulse response time duration. In PuCT, the sample is excited with a coded excitation of duration $T$ and thermograms are collected for an overall time duration of $T+T_{h}$. An estimated impulse response of duration $T_{h}$ is retrieved after performing the $\mathrm{PuC}$ algorithm (i.e. the duration of the equivalent $\mathrm{PT}$ analysis) [19,36].

$\mathrm{PuC}$ requires further processing to achieve an estimate $\tilde{h}(t)$ of the impulse response $h(t)$, the quality of the estimation depending strictly on both the correct implementation of the $\mathrm{PuC}$ algorithm and on the correct design of the coded signal [37]. The working principle of the $\mathrm{PuC}$ technique is sketched in Fig.3 (b). Given a coded excitation $s(t)$ of duration $T$ and bandwidth $B$, and another signal $\Psi(t)$, the so-called matched filter, such that their convolution "**" approximates the Dirac's Delta function $\delta(t)$ as:

$$
s(t) * \Psi(t)=\tilde{\delta}(t) \approx \delta(t)
$$

Then an estimate $\tilde{h}(t)$ of the $h(t)$ is obtained by convolving the recorded output signal $y(t)$ with the matched filter $\Psi(t)$. The process is mathematically shown in Eq. (5) for a single pixel of the acquired thermograms, in presence of an Additive-White-Gaussian-Noise $e(t)$, which is uncorrelated with $\Psi(t)$. By convolving the output signal $y(t)$ with the matched filter $\Psi(t)$, the impulse response can be obtained as:

$$
\tilde{h}(t)=y(t) * \Psi(t)=h(t) *{ }_{=}^{s(t) * \Psi(t)}+e(t) * \Psi(t)=h(t) * \tilde{\delta}(t)+\tilde{e}(t) \approx h(t)+\tilde{e}(t)
$$

As an advantage in $\mathrm{PuC}$, the impulse response can be estimated by delivering energy to the system in a significantly longer time with lower peak power compared to PT, hence avoiding possible thermal shocks in fragile materials and allowing the use of relatively-cheap heating source such as commercial LED chips. In this way, it is possible to provide more energy, and hence to increase the SNR and detectability of eddy current thermography system. The SNR gain is proportional to the $T \times B$ product, i.e. it can be enhanced almost arbitrarily by increasing either the time duration or the bandwidth of the coded waveform. It should be also noted that the limited $T \times B$ product of practically-employed coded signals results in an $\tilde{h}(t)$ always affected by the so-called "side-lobes", i.e. any local maxima of the $\tilde{h}(t)$ amplitude except the main lobe (main peak). This can be improved by a proper choice of the matched filter signal $\Psi(\mathrm{t})$ [54]. In this paper, $s(t)$ is a Barker Code (BC) of order equal to 13 and the matched filter $\Psi(\mathrm{t})$ has been chosen simply to be the time-reversed sequence of the input coded signal $s(-t)$ [55]. Details about the $\mathrm{BC}$ signal and how to implement it in ECPuCT will be shown in the next section. A thorough analysis of $\mathrm{PuC}$ lies beyond the scope of this work. A detailed discussion of PuC about coded waveforms implementation can be found in paper by Hutchins et al. [39]. 


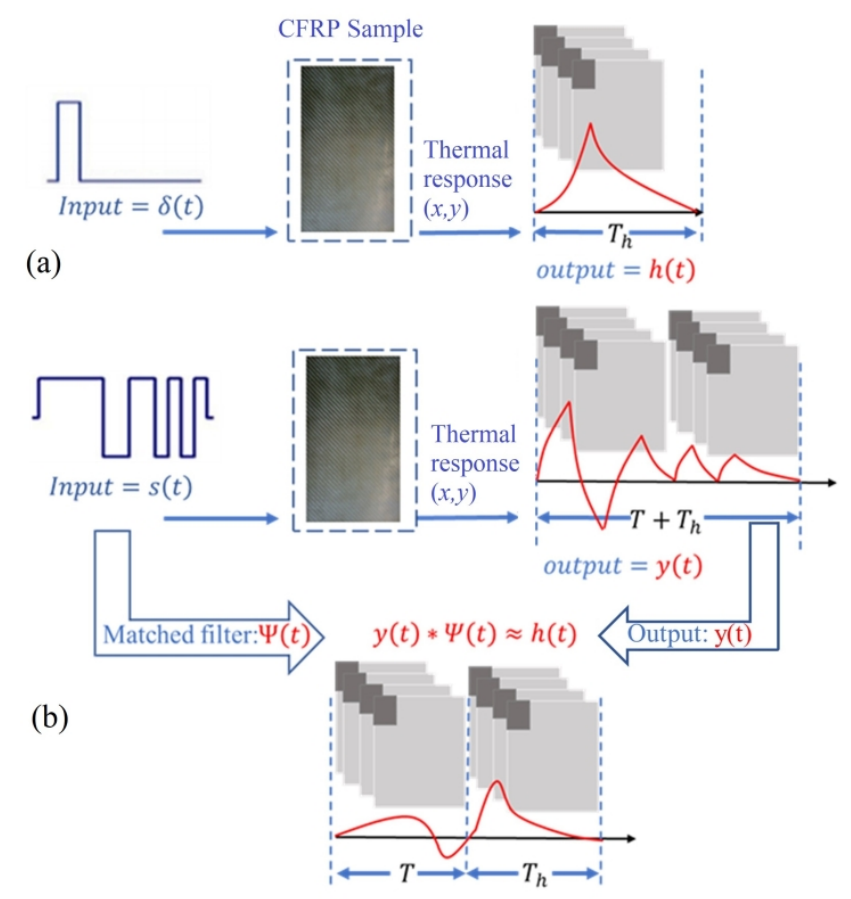

Fig. 3 Estimation of impulse response: (a) single pulse excitation, (b) Process of impulse response calculation

\subsection{Impulse response feature extraction process}

This section will give detailed explanation of the process depicted in block 4 of Fig.1. After calculating estimated impulse response $\tilde{h}(t)$, the K-PCA method is applied to locate the delaminated area and select abnormal pixels for feature extraction. The crossing point between defective and non-defective areas are selected for depth evaluation, whose process is presented in Fig 4.

Two different features including crossing point and skewness are extracted based on the selected impulse response. The crossing point is defined as the first cross point of the impulse response curves onto the defective area and non-defective area. The specific calculation of skewness to characterize the impulse responses' shape is presented in [56]. To calculate the features, the first 200 frames of $T_{h}$ were used, which is shown in fig. 4 and fig. 10 . The reason for using this period is that previous studies $[19,36]$ has shown that the range of interest of the collected impulse response is limited to less than 10 seconds for reflection mode PuCT analysis. Therefore, 200 frames have been here selected for the feature extraction in transmission mode, which corresponds to 4 seconds of diffusion time when an acquisition rate of 50 Frames Per Second (FPS) is employed. Moreover, side lobes affect the estimated impulse response would otherwise have a negative effect on the feature's extraction performance, e.g. reducing the sensitivity of proposed feature for depth evaluation.

The novel thermal pattern enhancement method is based on K-PCA method. Compared to the traditional PCA imaging, this method considers the impulse response of each pixel rather than each image in the thermal video as independent variable. Each extracted PC is a linear combination of the original impulse response and they form the basis of the respective vector space, arranged in order of decreasing variance. Thus, the first several PCs carry the most information regarding the original data [55].

To gain insight on how K-PCA is applied here for enhancing ECPuCT data, the implementation of the enhancement method is schematically depicted in Fig.4 (b) and mathematically introduced here below. Considering the calculated impulse response $\tilde{h}$ 's retrieved pixelwise by exploiting the procedure described in Eq.(5) being reshaped as:

$$
\left[\tilde{h}_{1}, \tilde{h}_{2}, \ldots, \tilde{h}_{M-1}, \tilde{h}_{M}\right]
$$


where $M$ is equal to $N_{x} \times N_{y}$ denotes the total number of $x$ and $y$ pixel of the acquired IR thermograms. Thus, the reshaped data can be recognized as a matrix having dimension of $Q \times M$, where $Q$ denotes the number of frames recorded by the IR camera, i.e. $T_{h} \times F P S$. By using the kernel method, the impulse response is projected to kernel space $\phi$, thus obtaining the kernel matrix $K(i, j)$ as:

$$
K(i, j)=\frac{1}{M} \sum_{i=1}^{M}\left(\phi\left(\tilde{h}_{i}\right)-\frac{1}{M} \sum_{j=1}^{M} \phi\left(\tilde{h}_{j}\right)\right)\left(\phi\left(\tilde{h}_{i}\right)-\frac{1}{M} \sum_{j=1}^{M} \phi\left(\tilde{h}_{j}\right)\right)^{\mathrm{T}}
$$

where $\phi$ is Gaussian kernel function, defined as Eq.(8):

$$
\phi\left(\tilde{h}_{i}^{1}, \tilde{h}_{i}^{2}\right)=\exp \left(\frac{\left\|\tilde{h}_{i}^{1}-\tilde{h}_{i}^{2}\right\|_{2}^{2}}{2 \sigma^{2}}\right)
$$

The kernel matrix $K(i, j)$ of Eq. (7), can be simply named as $K$. The eigenvector $\alpha$ of $K$ can be obtained as:

$$
\lambda_{i} \alpha_{i}=K \alpha_{i}
$$

Based on the obtained eigenvectors $\alpha_{i}$, the enhanced thermal pattern can be projected as:

$$
H_{\text {enhanced }}=\left[\alpha_{1}, \ldots, \alpha_{T}\right] H_{\text {original }}^{\mathrm{T}}
$$

where $H_{\text {enhanced }}$ contains different extracted thermal patterns.

After obtaining different thermal patterns projected by different PCs, in this paper PC2 and PC3 are exploited for differential computation as shown in Fig.12 to enhance the delaminated

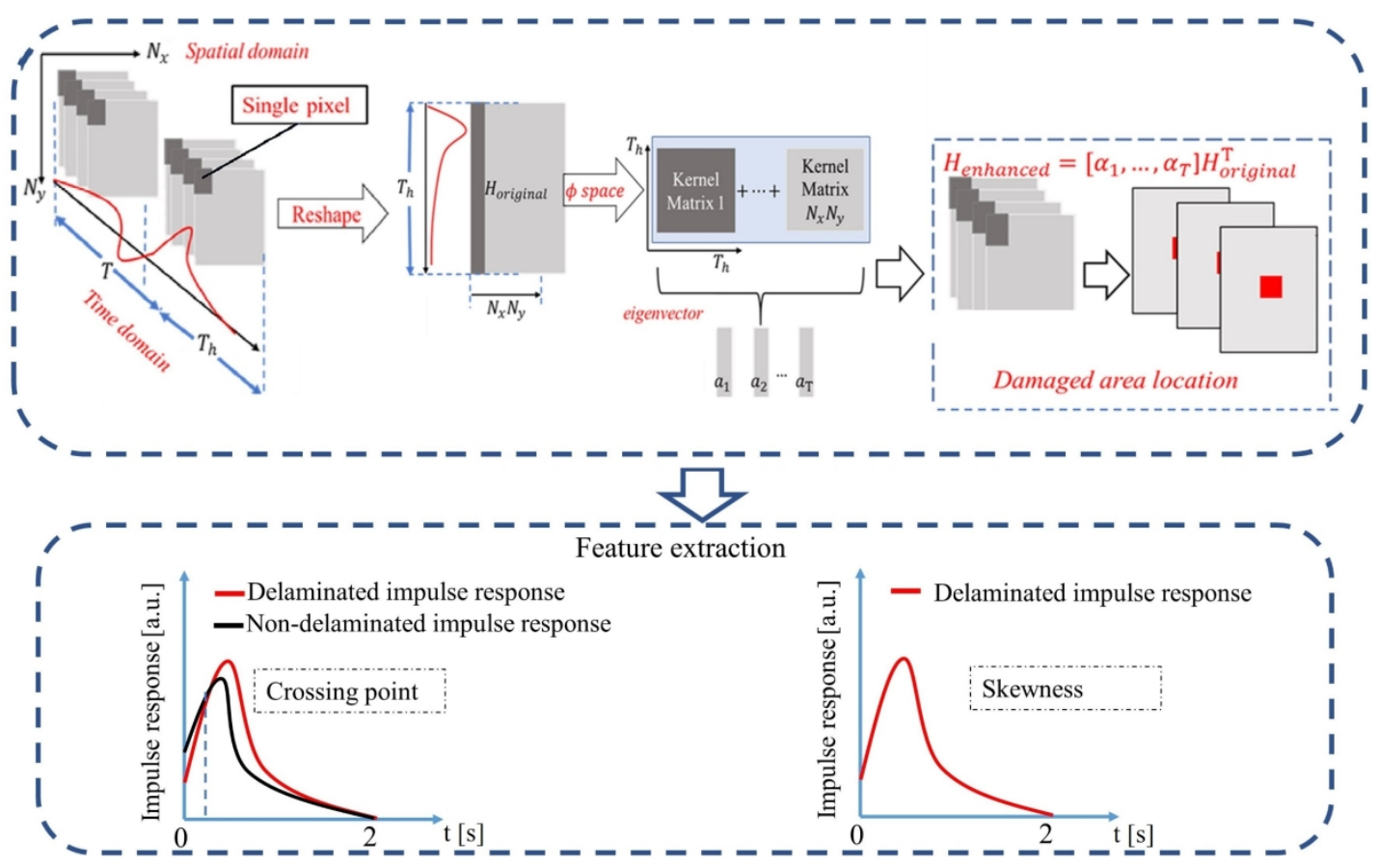

Fig. 4 Block diagram describing the implementation of the proposed feature extraction process.

area, which will be discussed in section 4.2.

\section{Experimental setup of eddy current pulse compression thermography}

\subsection{Barker code implementation in eddy current pulse compression thermography}


This section describes the process of the block 1 in Fig.1, in which the coded signal employed for modulating the induction heating system on/off state was a bipolar $\mathrm{BC}$ with a bit length of 13. However, the $\mathrm{BC}$ code is not employed in its original bit version in $\mathrm{PuCT}$ application. In fact, each " 1 " and "- 1 " of the original BC code is padded with a series of "1" or "-1" respectively to allow the heat source spreading enough energy toward the SUT. In addition, changing the single bit duration varies the frequency spectrum of the resulting $\mathrm{BC}$ modulated heating stimulus. This means that a proper design of the $\mathrm{BC}$ code leads to the onset of thermal waves having a desired yet needed thermal diffusion lengths $\mu$, allowing defects buried at different depths to be detected. Fig.5(a) shows the employed BC signal in which, each single bit lasts one second at the chosen FPS (50 frames per second), whilst its thermal frequency spectrum is depicted in Fig.5 (b) The thermal diffusion lengths $\mu$ can be calculated from Eq.(2), by considering both the diffusivity $\alpha$, and the frequency $f$ values of the modulated thermal frequency spectrum. For the chosen $\mathrm{BC}$ the maximum heat emission occurs at $0.50 \mathrm{~Hz}$.

In the implementation of $\mathrm{ECPuCT}$, the chosen $\mathrm{BC}$ modulates the induction heating unit, i.e. the on/off time instant at which a current $I$ of given amplitude $A m p$ and frequency $f_{\text {carrier }}$ flows within the coil. Fig. 6 shows a sketch of the modulated EC signal by BC in ECPuCT.
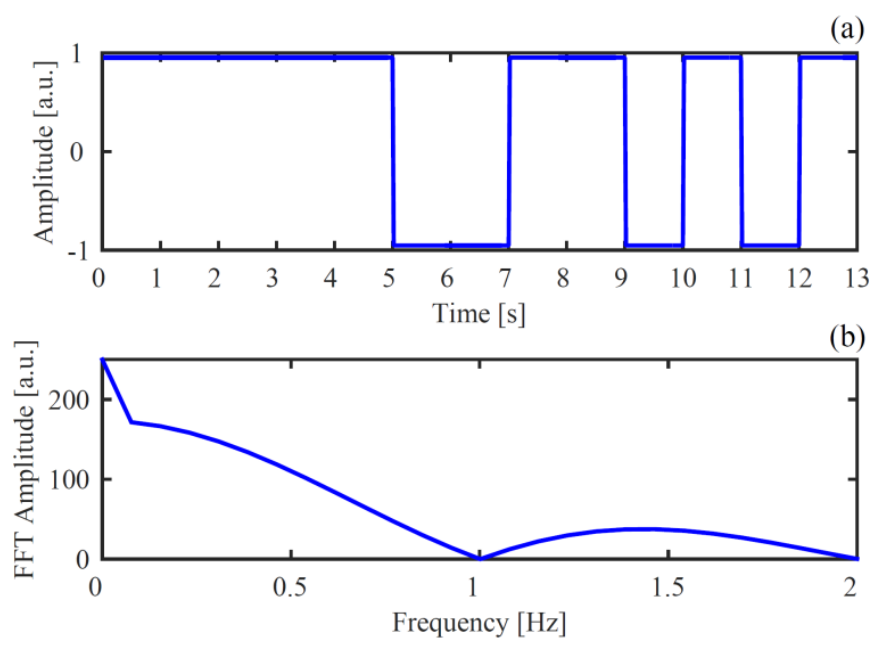

Fig. 5 Barker Code signal as implemented

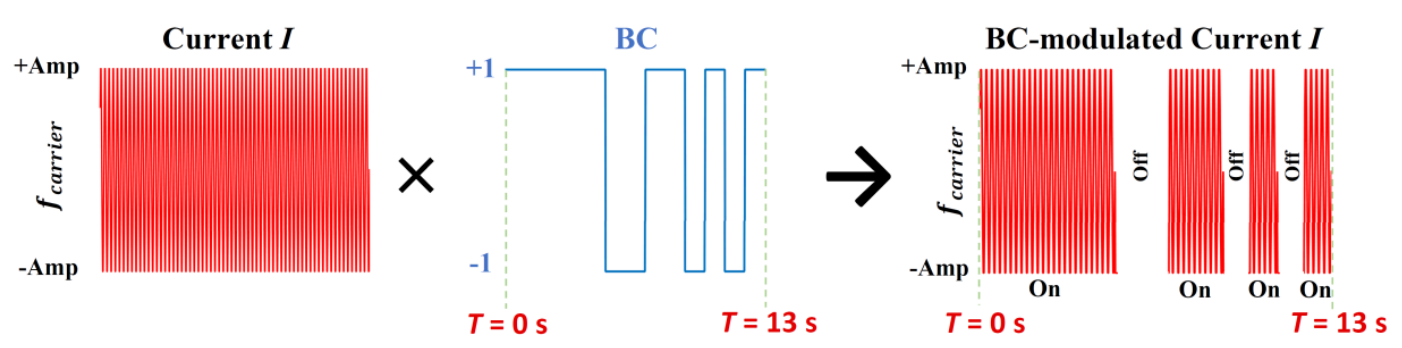

Fig. 6 A sketch of the modulated EC signal by Barker Code in ECPuCT used in the study

\subsection{Eddy current pulse compression thermography setup}

The ECPuCT system diagram is illustrated in Fig.7 (a). Signal generator is used to send both the $\mathrm{BC}$ modulating signal to the induction heating coil and a reference clock trigger to the IR camera to acquire thermograms at 50 FPS. A Cheltenham EasyHeat 224 Induction heating unit is used for exciting the coil with a maximum excitation power and current values of $2.40 \mathrm{~kW}$ and 400 A respectively with tunable $f_{\text {carrier }}$ within 150 to $400 \mathrm{kHz}$. For the reported experimental results, values of excitation current $I$ equal to $40 \mathrm{~A}$ and $f_{\text {carrier }}$ of $240 \mathrm{kHz}$ were selected to avoid eventual damage on the CFRP sample due to the long excitation time using $\mathrm{BC}$ signal. Fig.7 (b) shows a picture of the employed rectangular coil made from high- 
conductivity hollow copper tube with the outer and inner diameters of $6.35 \mathrm{~mm}$ and $4.00 \mathrm{~mm}$ respectively. Water cooling was implemented to the coil and the lift-off maintained at $3.00 \mathrm{~mm}$ from the SUT surface to ensure the volumetric heating of the sample. Only one side of the coil is selected as linear coil to introduce parallel eddy currents in the direction of maximum conductivity in the sample, the length of this side is $9.30 \mathrm{~mm}$. IR camera was the FLIR SC655, equipped with an un-cooled microbolometer detector array with the resolution of $640 \times 480$ pixels, the spectral range of $7.5-14.0 \mu \mathrm{m}$ and NETD $<30 \mathrm{mK}$. The IR camera records the surface temperature distribution of the $13 \mathrm{~s} \mathrm{BC}$ as well as additional 30 seconds of cooling period [19] with a frame rate of $50 \mathrm{~Hz}$, i.e. $50 \mathrm{FPS}$. Finally, captured thermal videos were transmitted to a $\mathrm{PC}$ for visualization and postprocessing, including signal pre-process, PuC, thermal pattern enhancement and depth feature extraction.

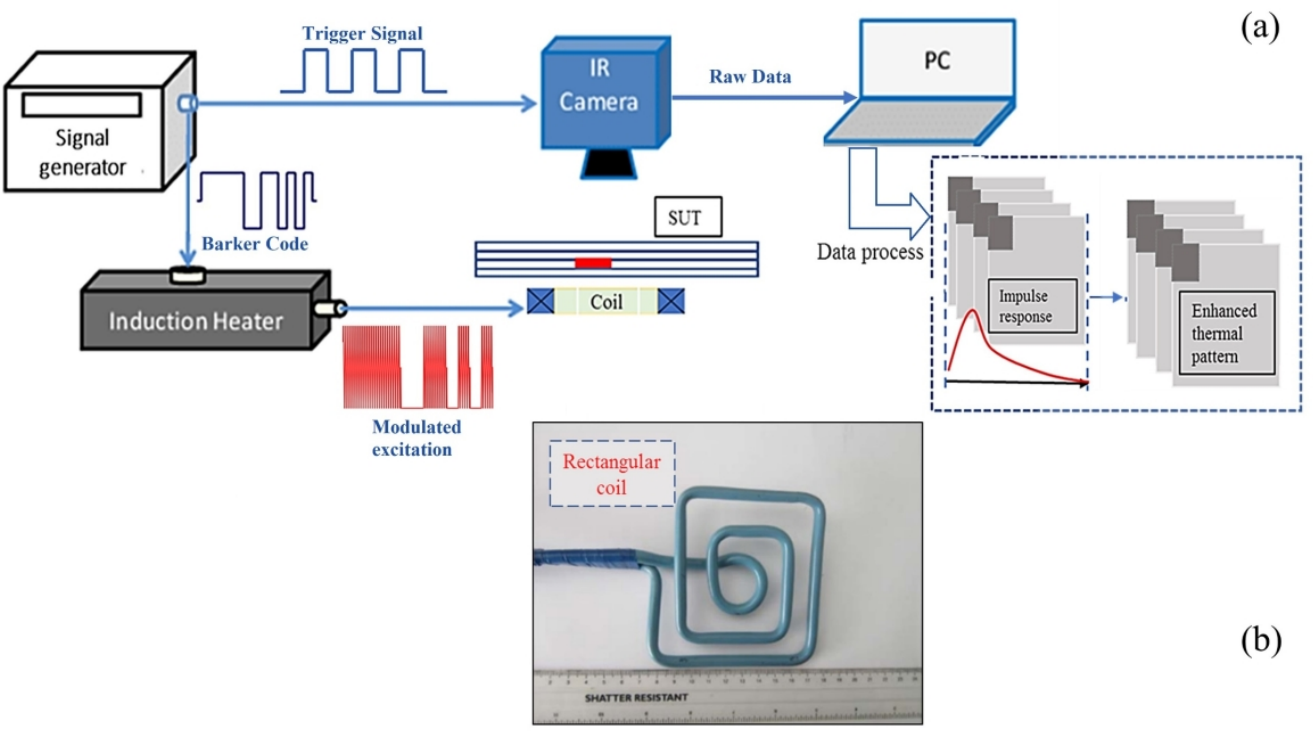

Fig. 7 Experimental setup: (a) system block diagram, (b) detail of the employed rectangular coil

\subsection{Sample under test}

The CFRP laminate sample was realized at University of Perugia [19] and contains twelve plies of carbon fiber fabric with an areal density of $0.20 \mathrm{~g} / \mathrm{m}^{3}$. Its lateral dimensions are 240 $\mathrm{mm} \times 200 \mathrm{~mm}$ for an overall thickness of $\sim 2.80 \mathrm{~mm}$. The fibers orientations are $0^{\circ}$ and $90^{\circ}$ and the matrix is an Epoxy Resin RIM 935. The laminate was made by vacuum assisted resin infusion and it was cured at room temperature and postured at $110^{\circ} \mathrm{C}$ for two hours to obtain a fully cured system. The artificial delamination defects were realized by inserting thin square pieces of Teflon tape having lateral dimensions of $20.00 \mathrm{~mm} \times 20.00 \mathrm{~mm}$ and thickness equal to $75.00 \mu \mathrm{m}$ between the plies as shown in Fig.8 (a). Nine artificial defects were inserted at increasing depths: defect $\# 1$, the shallowest, was placed under the $2^{\text {nd }}$ ply at a depth $d \sim 0.46 \mathrm{~mm}$ and defect \#9, the deepest, was under the $10^{\text {th }}$ ply at a depth of $d \sim 2.30 \mathrm{~mm}$ in the sample as shown in Fig. 8 (b) 


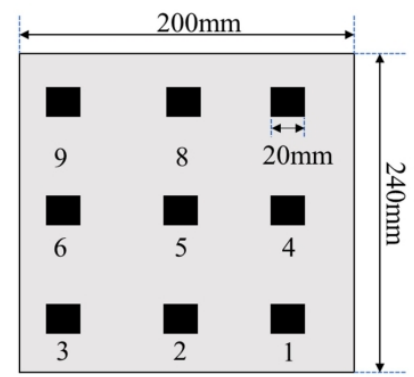

(a)

(b)

Each ply:0.23mm

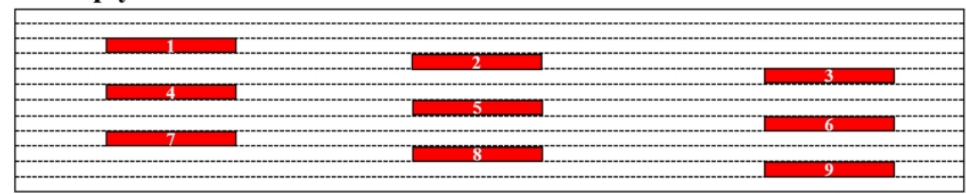

Fig.8 Schematic diagram of SUT: (a) plan view, (b) cross section view

\section{Results and analysis}

\subsection{Process of obtaining estimated impulse response}

As discussed in Section 3.1, the BC excitation is applied and raw data are recorded. As block 2 of Fig. 1 shows, the raw acquired data must be pre-processed before successfully applying the $\mathrm{PuC}$ algorithm to obtain estimated impulse response: the raw acquired data contains (i) noise generated during the signal acquisition process and (ii) an additional step-heating contribution due to the monopolar heating source that must be removed.

Regarding point (i), although the $\mathrm{PuC}$ algorithm acts as a low-pass filter as it is based on the convolution with a bandlimited matched filter signal $\Psi(t)$ (see Eq.(5)), it is crucial to minimize the noise to ensure a successful feature extraction of delamination depth. To this aim, wavelet analysis is acknowledged as the most effective method for de-noising non-stationary thermal signals [57]. By using the multi-resolution and self-similarity characteristics of wavelet analysis, the pseudo-white noise in non-stationary thermal signals can be eliminated.

Regarding point (ii), Silipigni et al. [19] presented different de-trending algorithms and polynomial functions for LED-stimulated PuCT application. Their proposed non-linear fitting function is here used. Fig.9 (a) shows the denoised thermal signal together with non-linear fitting function obtained for a single pixel, whilst Fig.9 (b) depicts the same signal after stepheating component removal, thus ready for PuC.

As discussed in Section 2.2, Fig.10 shows examples of the impulse responses obtained for a single pixel after PuC, hence obtained by convolving the signal depicted in Fig.9 (b) with the matched filter $\Psi(\mathrm{t})$, both for a $\mathrm{BC}$ retrieved raw signal and for a $\mathrm{BC}$ de-trended and denoised raw signal. It can be noticed that the signal maximum amplitude is reduced when applying denoising due to the elimination of non-stationary thermal noise, but the smoothness of the impulse response after $\mathrm{PuC}$ is increased with respect to one obtained directly from the unfiltered raw data. Fig. 10 depicts a sample impulse response of a single pixel, it can be observed that the level of the sidelobe compared to the main lobe maximum amplitude is $\sim 0.4$. This may affect the process of the feature extraction performance. Therefore, as explained in Section 2.3, the signal has been truncated from 0 to 200 frames of the corresponding $T_{h}$. 
(a)
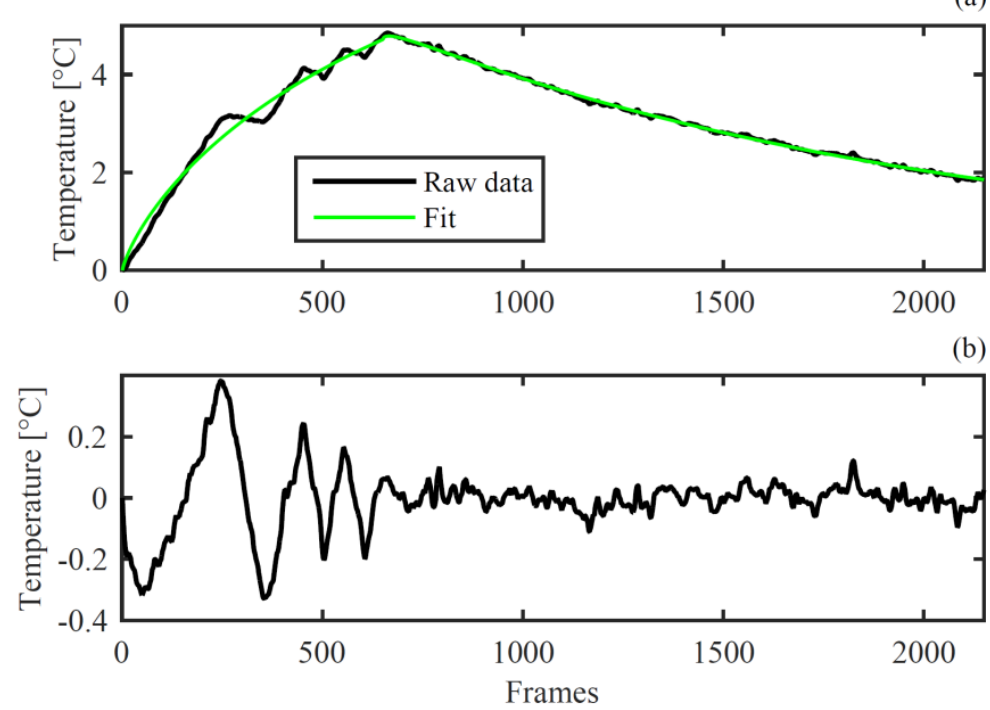

Fig. 9 Computation of de-trended signal for PuC: (a) BC raw data and non-linear fitting function; (b) De-trended signal

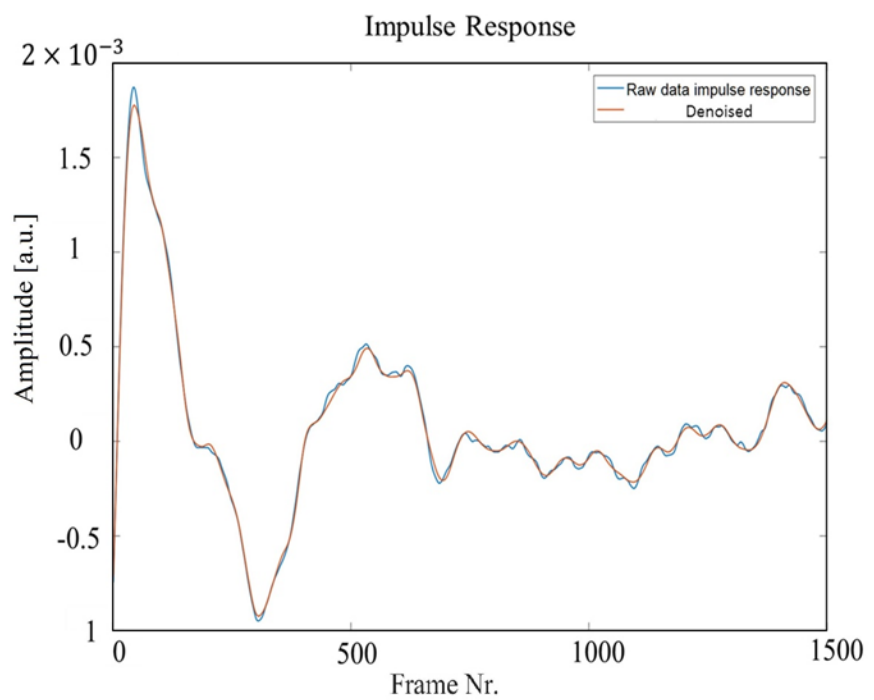

Fig. 10 Comparison of one pixel's raw and denoised impulse response

\subsection{Delaminated area location by Kernel-PCA}

As illustrated in block 4 of Fig.1, the K-PCA techniques is applied to enhance the delaminated area. In this section, detailed implementation will be introduced.

The impulse response $\tilde{h}(t)$ is a sequence of 1500 frames, i.e. 30 seconds corresponding to the whole $T_{h}$. However, when it comes to visualize the delamination, most of the frames contribute little to the defect location. To solve this problem, the K-PCA method described in section 2.3 is applied to find and learn the mutual relation of impulse response in individual pixels. Thus, the pixelwise-retrieved $\tilde{h}(t)$ 's are embedded into a suitable high-dimensional feature space through Gaussian kernel function. In addition, the kernel method is also an efficient way to imply the nonlinear characteristics of the raw data.

Fig. 11 shows the extracted thermal pattern from the raw data. It is noted that first thermal pattern projected by $\mathrm{PC} 1$ summarizes the overall heating phenomena during experiment because the heating is conducted on one side of rectangular coil, which generates linear heating, thus the elliptical pattern on PC1 images corresponds to the heating area. The sensitivity of pattern 1 for visualizing defect is heavily reduced for increased delamination depth. This is 
because pattern 1 contains most of information about heating pattern, which might cover up the faithful defect information. It is observed that patterns 2 and 3 projected by PC2 and PC3 respectively obtain opposite profile or weighting for delamination area. Pattern 2 generally presents the delamination in lower contrast while pattern 3 shows higher contrast compared to the surrounding area. This is because the eigenvectors extracted were supposed to be orthogonal with each other in kernel space $\phi$. The indicated relation between pattern 2 and pattern 3 can enhance the detectability in delamination area through logic operation, which is shown in Fig.12. In addition, since the largest electric and thermal conductivity values are along the fiber orientation, the delamination as a thermal barrier, hinders the heat diffusion mainly in $0^{\circ}$ and $90^{\circ}$ directions, which makes the delamination edges hotter and the delamination region colder. As a result, the delamination and its edge have a large contrast in the thermal pattern 1. Based on this fact, the enhanced thermal pattern of the nine defects are presented in Fig.13, where the achieved higher contrast shows the profile of delamination. Compared to the results in [19], where defect $\# 9$ was not observable under flash thermography and LED-stimulated PuCT, the induced eddy current can still penetrate into $2.30 \mathrm{~mm}$ depth to generate detectable IR signature of the deepest delamination. Defect SNR is reduced after D5, where the square shape of delamination profile cannot be identified. However, the delaminated area can still be characterized by fiber structure in $0^{\circ}$ and $90^{\circ}$ from D6 D9 since induced eddy currents are parallel to $0^{\circ}$ and $90^{\circ}$, which have the largest electrical conductivity and thermal conductivity in SUT thus generating abnormal thermal wave with diffusion length $\mu$ longer than the delamination depth $d$.

Generally, artificial delamination in CFRP has been demonstrated to exhibit different patterns by K-PCA method. The enhanced pattern emphasizes the defect with a larger contrast in comparison to the sound regions and provides a guide on how to select the proper pixels or regions of impulse response to quantitively evaluate the different depths of delamination. In the Sections 4.3 and 4.4, the impulse response retrieved at the most abnormal pixels area are investigated for feature extraction. 

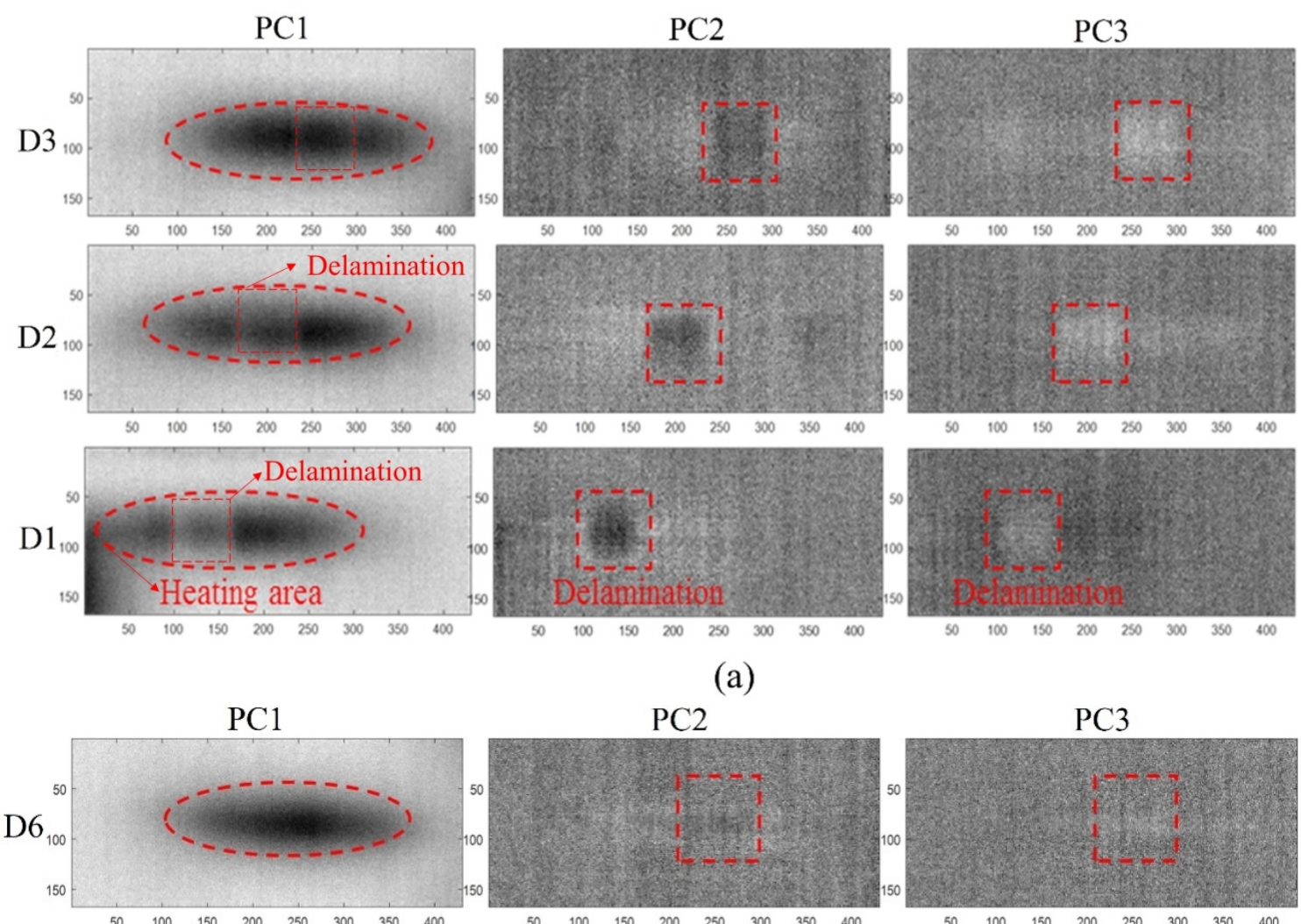

(a)
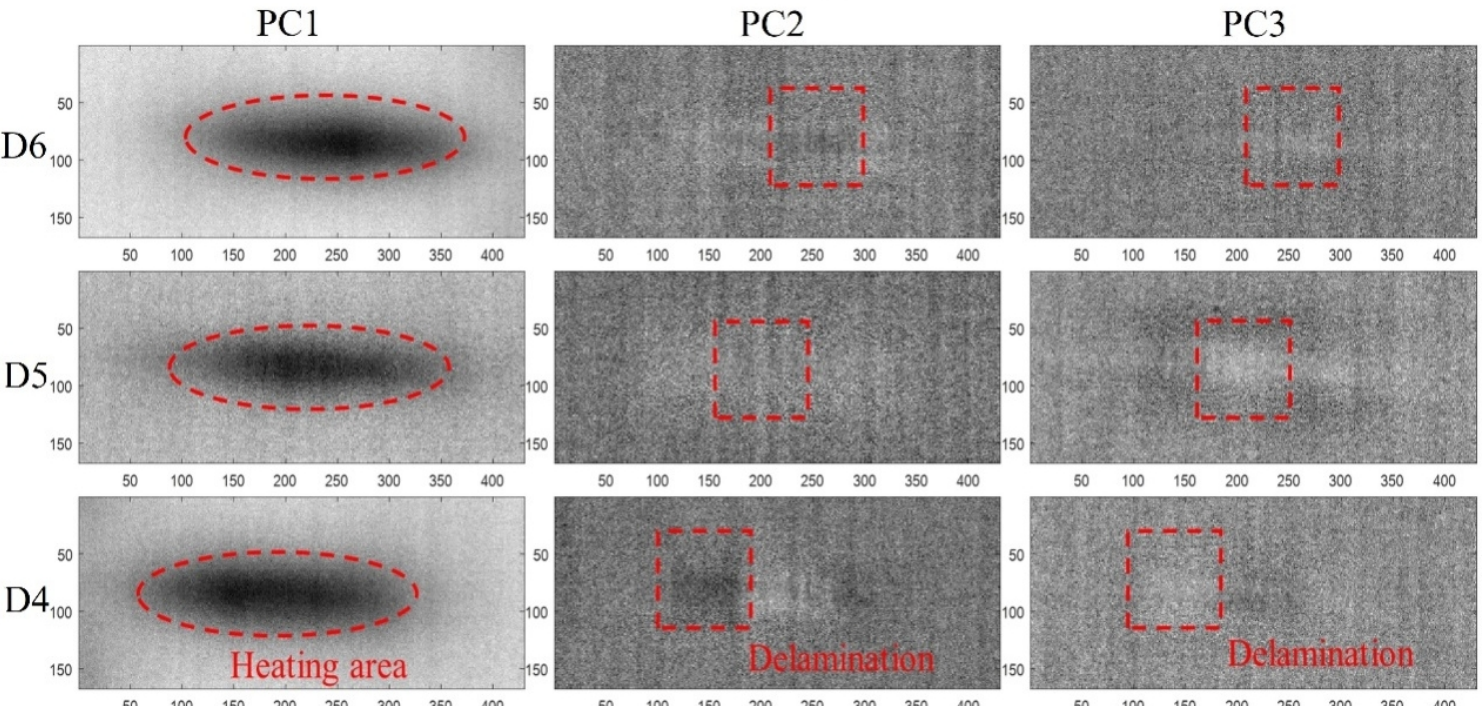

(b)
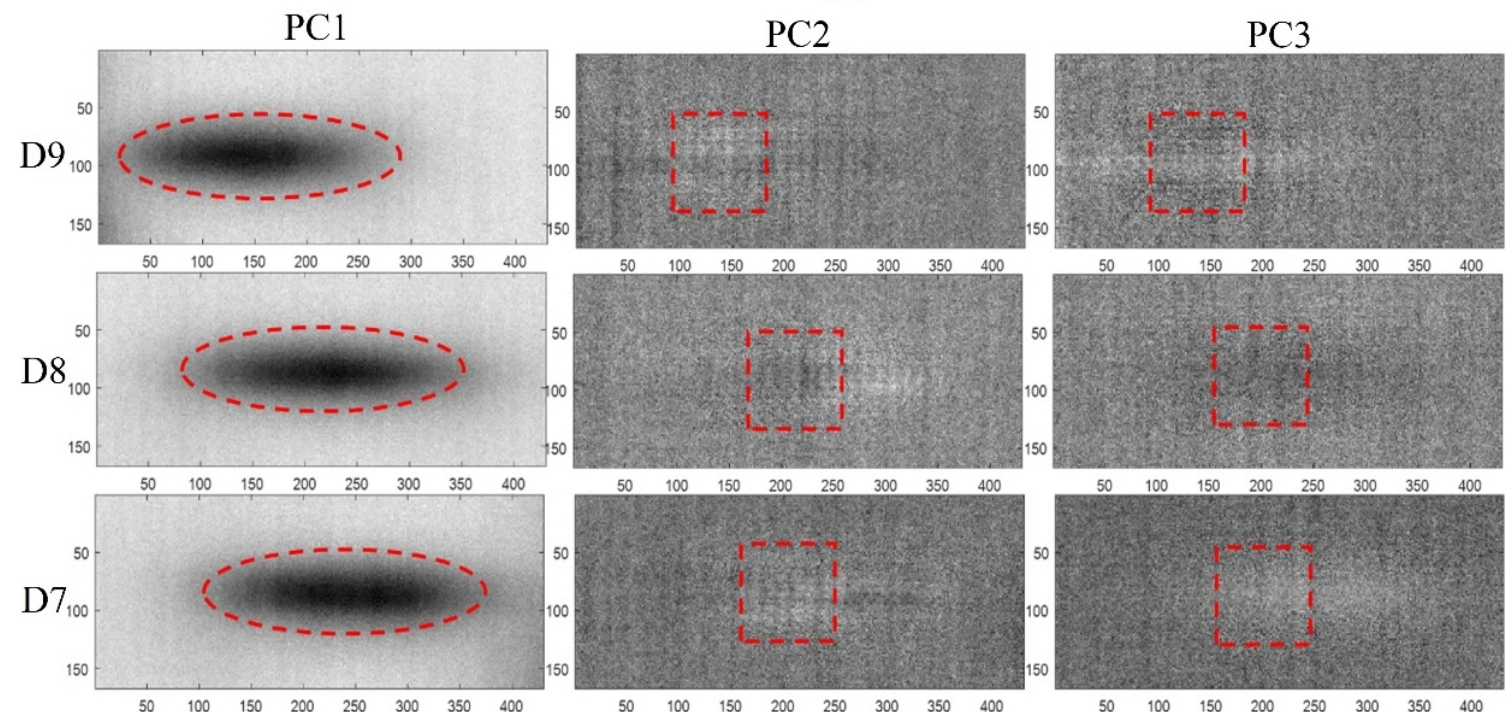

(c)

Fig.11 First three extracted thermal patterns: (a) D1, D2, D3, (b) D4, D5, D6, (c) D7, D8, D9 


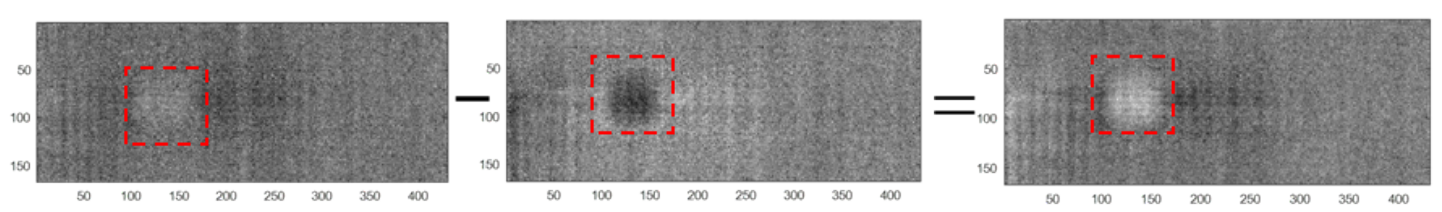

Fig. 12 Process of obtaining enhanced pattern by subtraction of PC2 and PC3 projected patterns
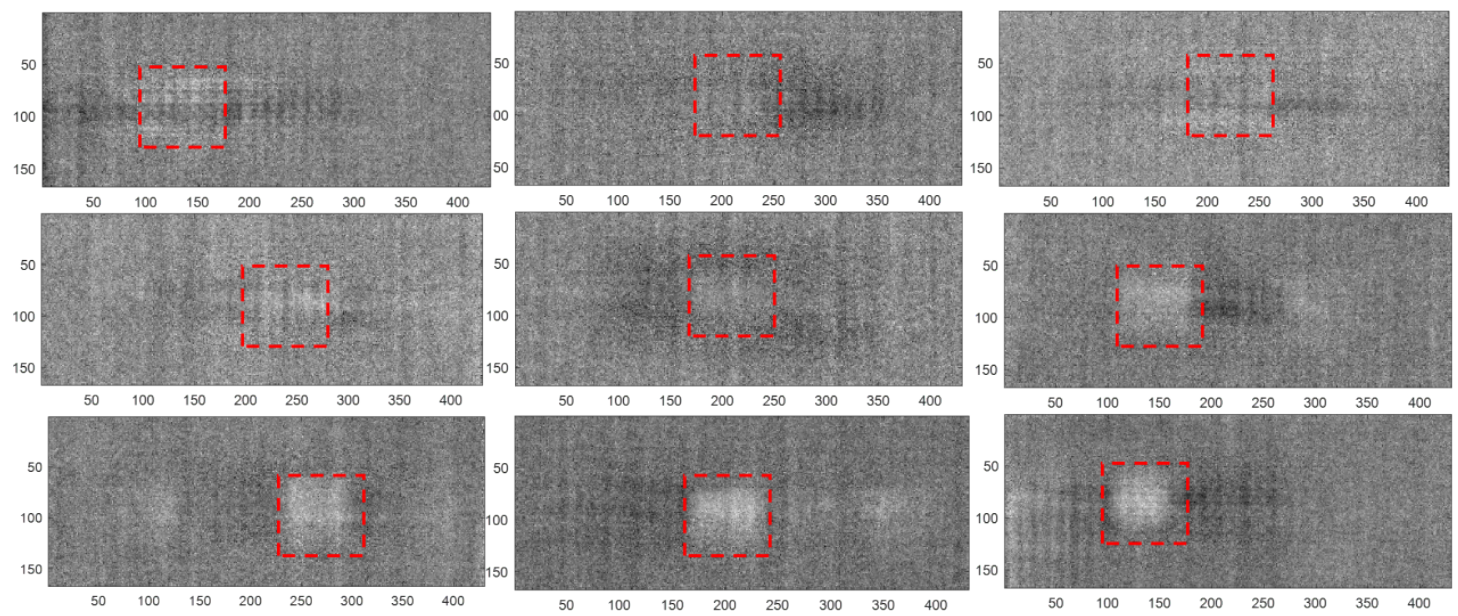

Fig. 13 Enhanced thermal pattern D1 D9

\subsection{Crossing point feature analysis}

As discussed in Section.4.2, the delamination ranging from $0.46 \mathrm{~mm}$ to $2.30 \mathrm{~mm}$ of depth can be visualized by enhanced thermal pattern method based on the impulse response. Moreover, to determine the delamination depth, the phase contrast between defected and nondefected areas can be exploited, by comparison of the obtained impulse responses based on PT theory [58]. The defected areas are detected and selected by K-PCA as shown in Fig.14 in line with the sample introduction in the Fig. 8.

Each defected and non-defected areas are formed by five lines of data in the center of higher contrast and lower contrast based on enhanced pattern. Based on the selected area, the comparison between impulse responses of defected and non-defected areas are presented in the plot series of Fig. 15. It can be noted that the impulse response curves behave similarly on the general trend for both the defective and the sound areas. Fig. 15 shows that the crossing point feature for the D1 and D2 defects (shallower ones) are in the heating part of the curve. According to [59] and Section 2 above, this can be related to the interaction of the eddy current's interaction with defect. However, the crossing points in defects D3 to D9 (deeper ones) are in the cooling stage of the impulse responses, which corresponds to the depth of the defect and the heat transfer phenomena as explained in Fig. 2. This means that deeper defects need longer time to be detected, as expected. Results showed in Fig. 17 demonstrates that the impulse response amplitude is less sensitive than the phase change of crossing point of defective and non-defective area. Thus, it is observed that the position of the first crossing point of impulse responses of defected and non-defected areas has a monotonic relationship with delamination depth, as shown in Fig.15 (labelled area) and Fig.16. This can serve as an excellent feature to quantify the delamination depth. From Fig.16, it can be noted that the feature based on the crossing point value follows a linear trend if three defects in a row over the SUT are considered, e.g. D1-D3 or D6-D9, which is due to the linear coil used in this experiment. In fact, the experimental conditions can be considered the same if defects are on the same line. Better results could be possibly obtained by achieving a more uniform heating through optimizing coil configuration.

However, after D6, the crossing pattern of defected and non-defected areas has been disturbed by noise as shown in Fig.15 (c). Moreover, extra crossing points are observed in D7, continuous equal value of defected and non-defected areas in D8 and initial disturbed crossing 
point in D9 are caused by the low SNR of original signal. Thus, despite the effectiveness of the denoised approach applied in this work, accuracy sizing of defect is still challenging to be extracted in D7 D9.

To validate this crossing point feature of impulse responses, the experiments were conducted again under reflection modes. The process of feature extraction for transmission and reflection modes is the same as illustrated of the Fig.4. It is observed in Fig.16 and Fig.17 (b) that the proposed features have monotonic relationships with delamination depth in reflection mode. Furthermore, the proposed feature in reflection mode holds better linearity and sensitivity as shown in Fig.17 (b) and better stability (less standard deviation error) as in Fig.16 (b) than transmission mode. In addition, compared with impulse responses in transmission mode in Fig.15, the response curves of the delaminated and the non-delaminated areas in reflection mode becomes divergent. These differences can also be investigated in the future.

To summarize, the proposed crossing point feature was validated in both transmission and reflection modes, so it can be concluded that the proposed feature can be used for quantifying CFRP delamination depth. The cross-point feature, i.e. cross-point time reflects the defect depths and the skin depths of eddy current in both transmission and reflection modes.
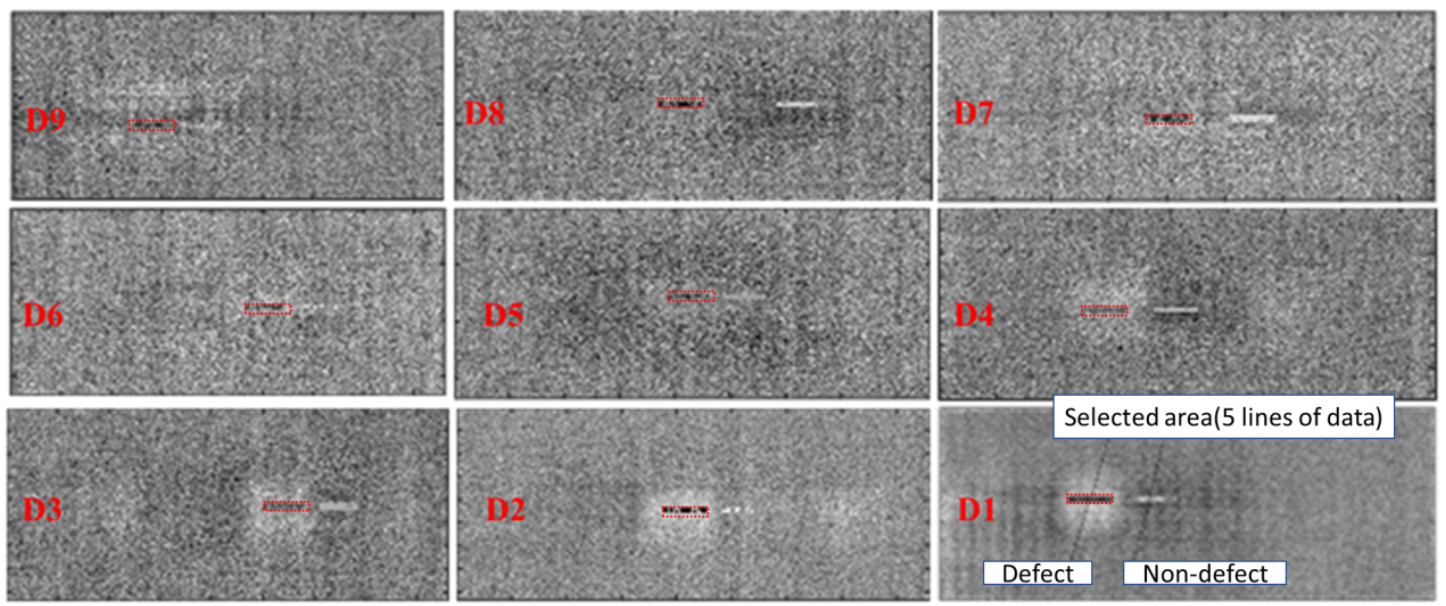

Fig. 14 Selected damaged area and non-damaged area based on KPCA enhanced pattern 

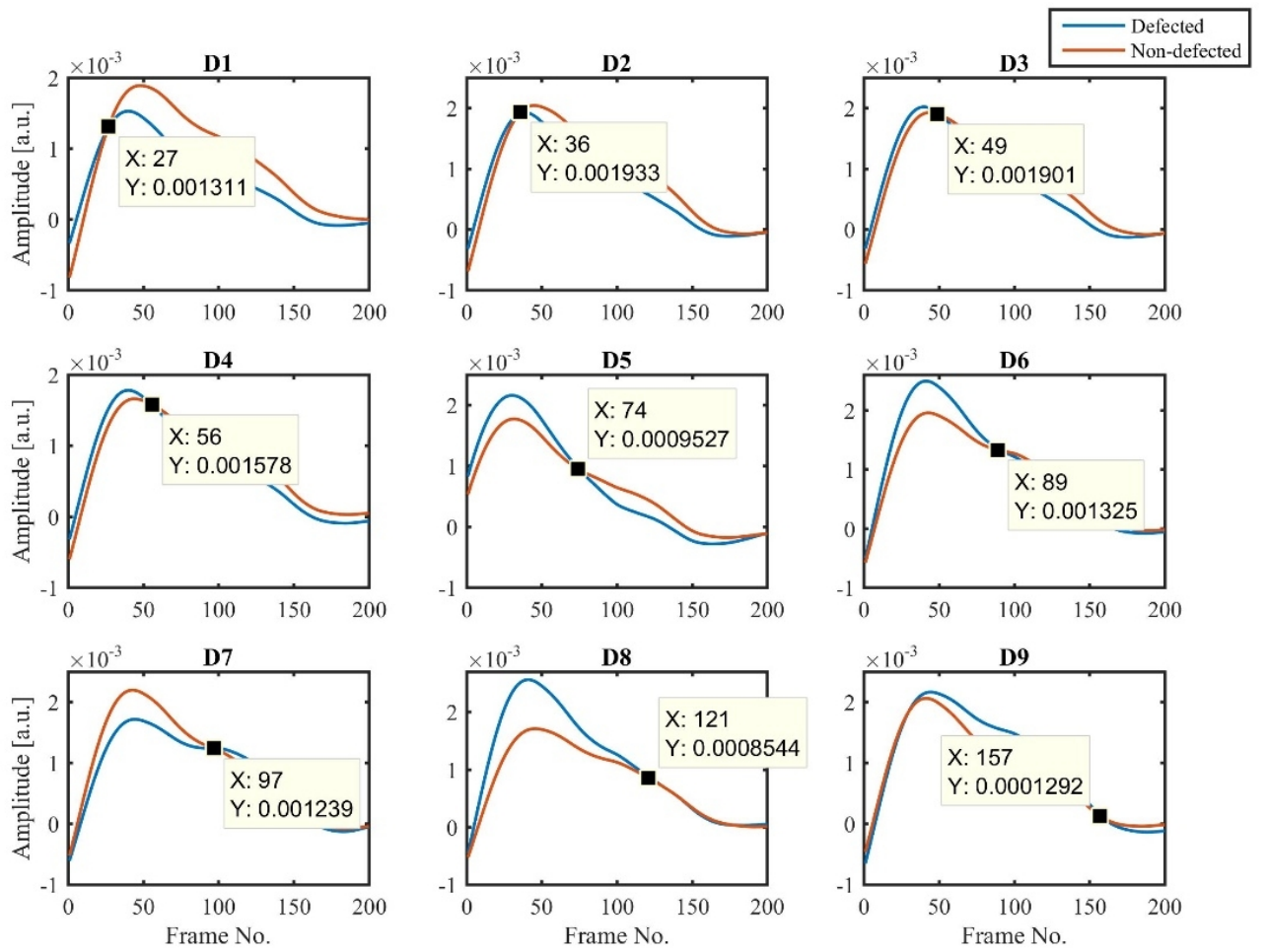

Fig. 15 Crossing point feature obtained from mean value of selected impulse responses in transmission mode
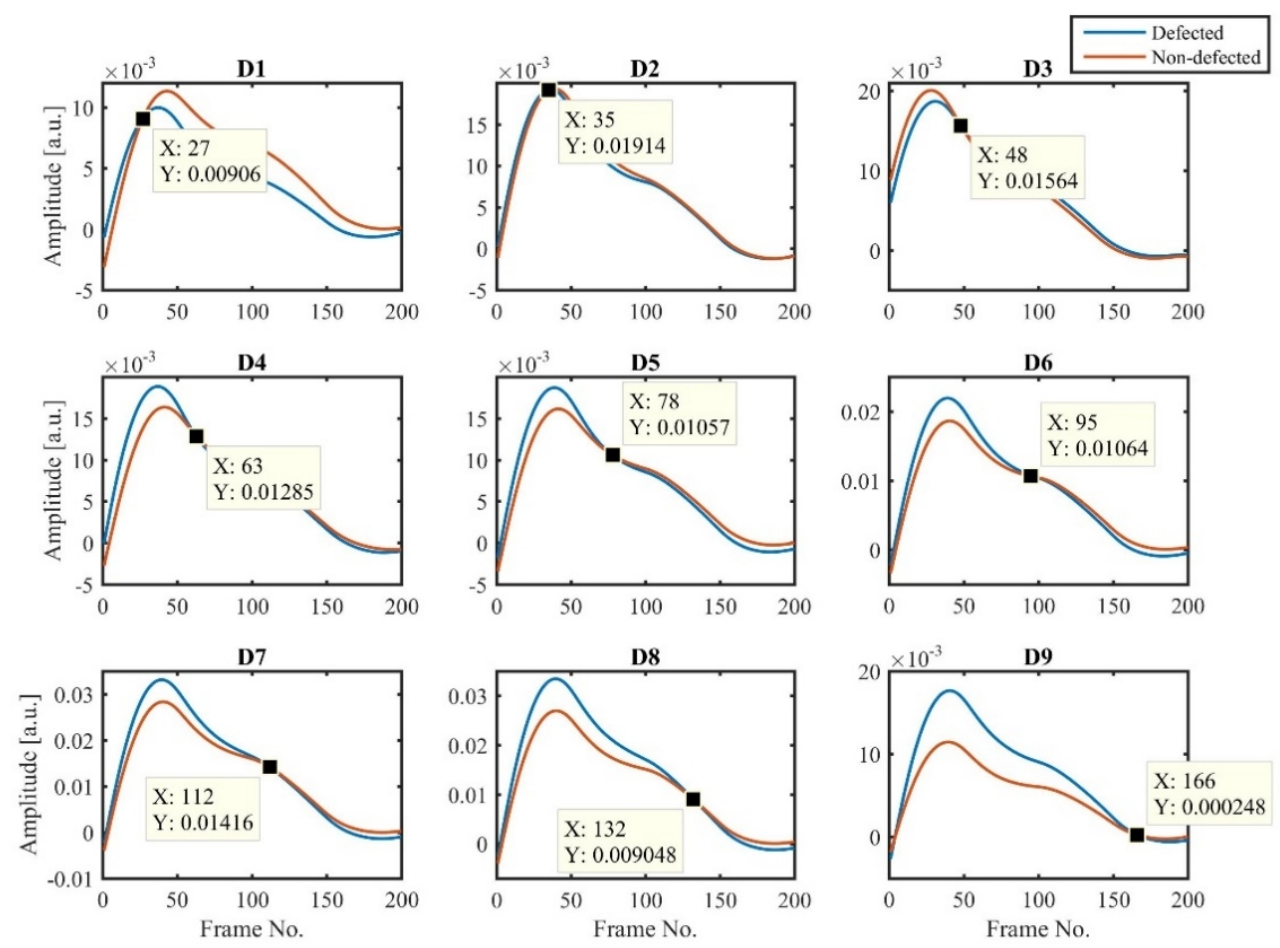

Fig. 16 Crossing point feature obtained from mean value of selected impulse responses in reflection mode 


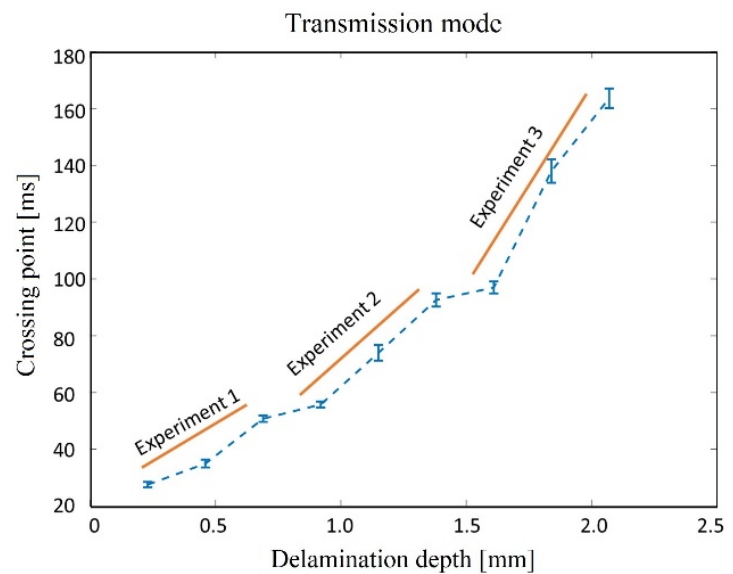

(a)

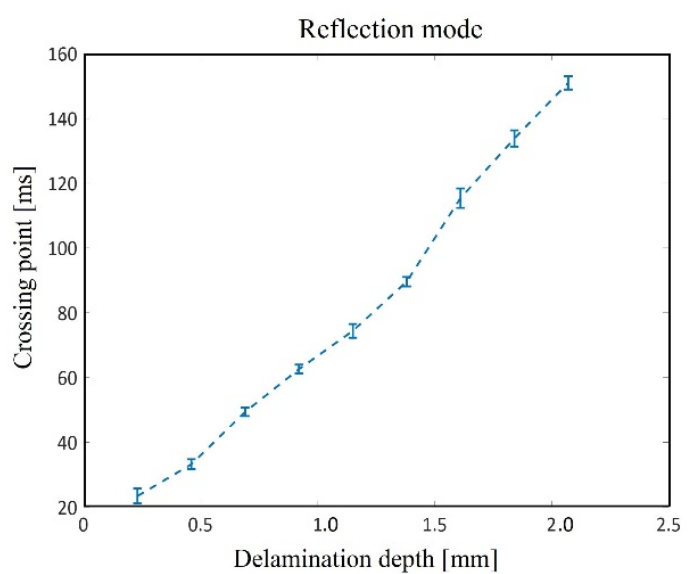

(b)

Fig. 17 Feature validation of crossing point with error bar versus delamination depth in:(a) transmission mode, (b) reflection mode

\subsection{Skewness feature analysis}

Based on the mathematical definition of skewness, this feature can be used to quantify the asymmetry of the considered data. In this section, the skewness is calculated on the major part of the impulse responses (first 200 frames of $T_{h}$ period). It is observed that positive skewness values are found for impulse responses as depicted in Fig.18. In Fig.18 (a), the skewness of data in transmission mode shows monotonic relationship with delamination depth because the impulse response curves of ninth defects are becoming more symmetrical along with the increase of delamination depth. However, in reflection mode, Fig.18 (b), this relationship becomes less monotonic than in transmission mode, which might be due to the texture influence on the selection of abnormal pixels. As a matter of fact, in reflection mode the IR camera is much closer to the coil than the transmission mode to maintain the same lift-off in two different modes. Thus, the fiber texture contribute in reflection mode is more evident than in transmission mode. Fig.19 presents the enhanced pattern of D1 by K-PCA in transmission and reflection modes. It is observed that fiber structure in reflection mode is more obvious than the transmission mode, which proves the idea that texture influence is more significant in reflection mode. The crossing point performance is also good in reflection mode, even with surface texture influence, as illustrated in Fig.17 (b).

Overall, the skewness of extracted impulse response can help characterize the delamination depth because of its monotonic relationship with delamination depth especially in transmission mode.

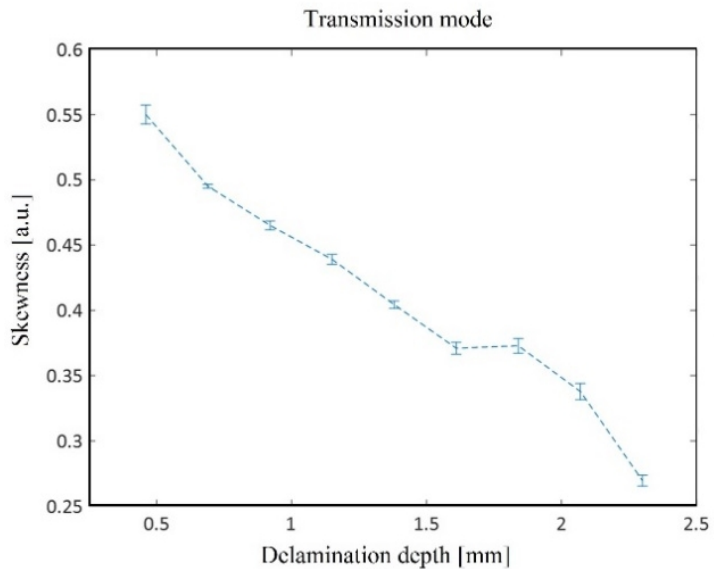

(a)

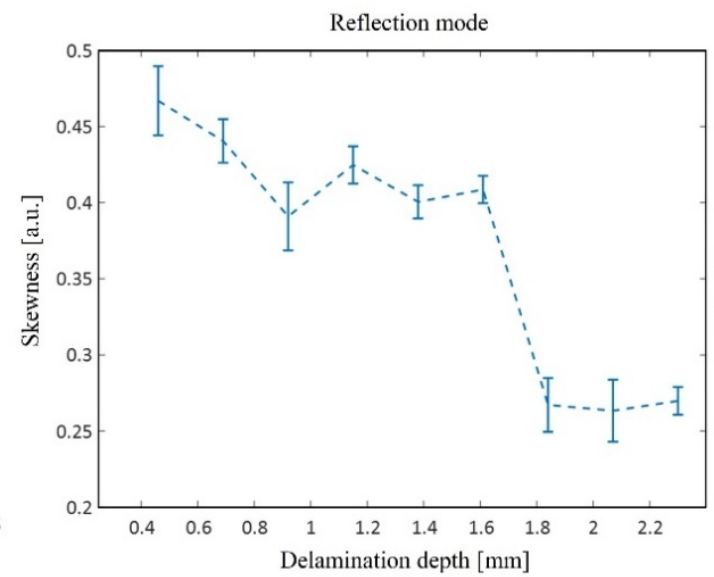

(b)

Fig.18 Error bar plot of the skewness features in different modes: (a) transmission mode,

(b) reflection mode 


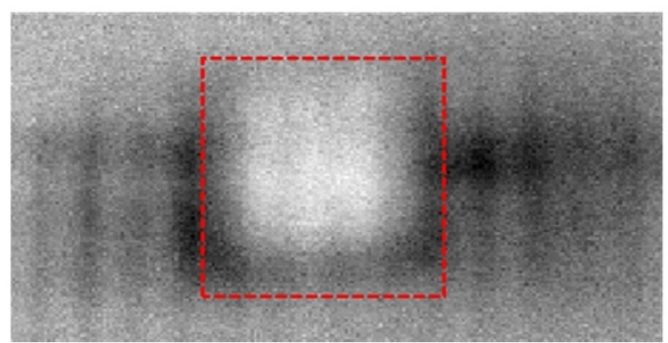

(a)

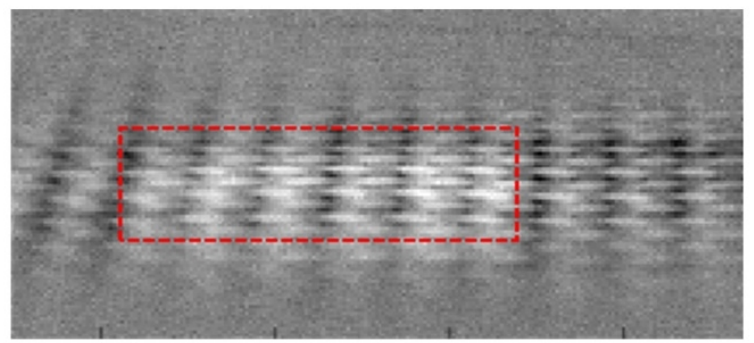

(b)

Fig.19 Enhanced pattern of defect \#1: (a) transmission mode, (b) reflection mode

\section{Conclusion and future work}

In this work, ECPuCT has been presented for characterization of delamination depth in CFRP laminates using different features comparison and validation including crossing point and skewness. The proposed features were verified through experimental studies under both transmission and reflection modes. The conclusions are as follows:

1) K-PCA method can well extract the meaningful pattern in the impulse response behavior as time elapses, helping thus in locating the delamination areas effectively. It was observed that the method can reveal the spatial pattern, which corresponds to the defect with enhanced contrast, hence improving defect detectability and noise reduction capability.

2) The proposed crossing point feature is extracted based on the crossing point of impulse response from defective and non-defective areas previously discriminated by K-PCA method. This feature has monotonic trend in both transmission and refection modes for delamination depths. The feature performance in term of linearity is better in reflection mode than in transmission mode.

3) Skewness feature of impulse response is also investigated. It illustrates the monotonic relationship with delamination depths in transmission mode as well. However, the monotonic relationship does not keep with delamination depths in reflection mode due to the influence of the texture structure. Compared with other features' performance in reflection mode, the feature of crossing point demonstrates more robustness against texture to ensure the stability in reflection mode.

In addition to more samples for evaluation, future work will also investigate:

1) The use of other coded signals and the optimization of Barker code single bit length for $\mathrm{ECPuCT}$ and further quantitative evaluation of texture influence on the proposed features.

2) The application of the proposed features of depth characterization on thermographic tomography and further investigation of different range of impulse responses for characterizing eddy current and thermal diffusion for evaluation of low energy impact damage and fiber breakage in composites materials.

\section{Acknowledgments}

This project is funded by the European Union's Horizon 2020 research and innovation programme under the Marie Skłodowska-Curie grant agreement No 722134 - NDTonAIR. The authors also would like to thank Prof. Luigi Torre and Dr. Roberto Petrucci from University of Perugia for the realization of the sample used in this work.

\section{References}

[1] Zhou A, Tam L-h, Yu Z, Lau D. Effect of moisture on the mechanical properties of CFRPwood composite: an experimental and atomistic investigation. Composites Part B: Engineering. 2015;71:63-73. 
[2] Mouritz A, Leong K, Herszberg I. A review of the effect of stitching on the in-plane mechanical properties of fibre-reinforced polymer composites. Composites Part A: applied science and manufacturing. 1997;28:979-91.

[3] Nakahata K, Ogi K, Namita T, Ohira K, Maruyama M, Shiina T. Photoacoustic microscopic imaging of surface and subsurface damages in CFRP. AIP Conference Proceedings: AIP Publishing; 2018. p. 180004.

[4] Gholizadeh S. A review of non-destructive testing methods of composite materials. Procedia Structural Integrity. 2016;1:50-7.

[5] Yang R, He Y. Optically and non-optically excited thermography for composites: A review. Infrared Physics \& Technology. 2016;75:26-50.

[6] Milovanović B, Banjad Pečur I. Review of active IR thermography for detection and characterization of defects in reinforced concrete. Journal of Imaging. 2016;2:11.

[7] Benítez HD, Ibarra-Castanedo C, Bendada A, Maldague X, Loaiza H, Caicedo E. Definition of a new thermal contrast and pulse correction for defect quantification in pulsed thermography. Infrared Physics \& Technology. 2008;51:160-7.

[8] Steinberger R, Leitão TV, Ladstätter E, Pinter G, Billinger W, Lang R. Infrared thermographic techniques for non-destructive damage characterization of carbon fibre reinforced polymers during tensile fatigue testing. International Journal of Fatigue. 2006;28:1340-7.

[9] Mendioroz A, Fuente-Dacal R, Apiñaniz E, Salazar A. Thermal diffusivity measurements of thin plates and filaments using lock-in thermography. Review of Scientific Instruments. 2009;80:074904.

[10] Sfarra S, Ibarra-Castanedo C, Paoletti D, Maldague X. Infrared vision inspection of cultural heritage objects from the city of L'Aquila, Italy and its surroundings. Materials Evaluation. 2013;71.

[11] Li K, Tian GY, Cheng L, Yin A, Cao W, Crichton S. State detection of bond wires in IGBT modules using eddy current pulsed thermography. IEEE Transactions on Power Electronics. 2014;29:5000-9.

[12] Weschenfelder AV, Maldague X, Rocha LM, Schaefer AL, Saucier L, Faucitano L. The use of infra-red thermography for pork quality prediction. Meat Sci. 2014;96:120-5.

[13] Maldague X. Theory and practice of infrared technology for nondestructive testing. 2001.

[14] Maierhofer C, Myrach P, Reischel M, Steinfurth H, Röllig M, Kunert M. Characterizing damage in CFRP structures using flash thermography in reflection and transmission configurations. Composites Part B: Engineering. 2014;57:35-46.

[15] Ibarra-Castanedo C, Sfarra S, Ambrosini D, Paoletti D, Bendada A, Maldague X. Diagnostics of panel paintings using holographic interferometry and pulsed thermography. Quantitative InfraRed Thermography Journal. 2010;7:85-114.

[16] Bendada A, Sfarra S, Ibarra C, Akhloufi M, Pradere C, Maldague X. Subsurface imaging for panel paintings inspection: a comparative study of the ultraviolet, the visible, the infrared and the terahertz spectra. Opto-Electronics Review. 2015;23:90-101.

[17] Bodnar J, Nicolas J, Candoré J, Detalle V. Non-destructive testing by infrared thermography under random excitation and ARMA analysis. International Journal of Thermophysics. 2012;33:2011-5.

[18] Schlichting J, Maierhofer C, Kreutzbruck M. Crack sizing by laser excited thermography. NDT \& E International. 2012;45:133-40.

[19] Silipigni G, Burrascano P, Hutchins DA, Laureti S, Petrucci R, Senni L, et al. Optimization of the pulse-compression technique applied to the infrared thermography nondestructive evaluation. NDT \& E International. 2017;87:100-10.

[20] Salazar A. Energy propagation of thermal waves. European journal of physics. 2006;27:1349.

[21] Gleiter A, Riegert G, Zweschper T, Busse G. Ultrasound lock-in thermography for advanced depth resolved defect selective imaging. Insight-Non-Destructive Testing and Condition Monitoring. 2007;49:272. 
[22] Wilson J, Tian G, Abidin I, Yang S, Almond D. Pulsed eddy current thermography: system development and evaluation. Insight-Non-Destructive Testing and Condition Monitoring. 2010;52:87-90.

[23] Palumbo D, Ancona F, Galietti U. Quantitative damage evaluation of composite materials with microwave thermographic technique: feasibility and new data analysis. Meccanica. 2015;50:443-59.

[24] Ren W, Liu J, Tian GY, Gao B, Cheng L, Yang H. Quantitative non-destructive evaluation method for impact damage using eddy current pulsed thermography. Composites Part B: Engineering. 2013;54:169-79.

[25] Cheng L, Gao B, Tian GY, Woo WL, Berthiau G. Impact damage detection and identification using eddy current pulsed thermography through integration of PCA and ICA. IEEE Sensors Journal. 2014;14:1655-63.

[26] Liang T, Ren W, Tian GY, Elradi M, Gao Y. Low energy impact damage detection in CFRP using eddy current pulsed thermography. Composite Structures. 2016;143:352-61.

[27] Cheng L, Tian GY. Comparison of nondestructive testing methods on detection of delaminations in composites. Journal of sensors. 2012;2012.

[28] Yang R, He Y. Eddy current pulsed phase thermography considering volumetric induction heating for delamination evaluation in carbon fiber reinforced polymers. Applied Physics Letters. 2015;106:234103.

[29] He Y, Yang R. Eddy current volume heating thermography and phase analysis for imaging characterization of interface delamination in CFRP. IEEE Transactions on Industrial Informatics. 2015;11:1287-97.

[30] Riegert G, Zweschper T, Busse G. Lockin thermography with eddy current excitation. Quantitative InfraRed Thermography Journal. 2004;1:21-32.

[31] Beuve S, Qin Z, Roger J-P, Holé S, Boué C. Open cracks depth sizing by multi-frequency laser stimulated lock-in thermography combined with image processing. Sensors and Actuators A: Physical. 2016;247:494-503.

[32] Maldague X, Marinetti S. Pulse phase infrared thermography. Journal of applied physics. 1996;79:2694-8.

[33] Mandelis A. Frequency Modulated (FM) Time Delay-Domain Thermal Wave Techniques, Instrumentation and Detection: A Review of the Emerging State of the Art in QNDE Applications. Review of Progress in Quantitative Nondestructive Evaluation: Springer; 1987. p. 799-806.

[34] Mulaveesala R, Venkata Ghali S. Coded excitation for infrared non-destructive testing of carbon fiber reinforced plastics. Review of Scientific Instruments. 2011;82:054902.

[35] Tabatabaei N, Mandelis A. Thermal-wave radar: A novel subsurface imaging modality with extended depth-resolution dynamic range. Review of Scientific Instruments. 2009;80:034902.

[36] Gong J, Liu J, Qin L, Wang Y. Investigation of carbon fiber reinforced polymer (CFRP) sheet with subsurface defects inspection using thermal-wave radar imaging (TWRI) based on the multi-transform technique. Ndt \& E International. 2014;62:130-6.

[37] Laureti S, Silipigni G, Senni L, Tomasello R, Burrascano P, Ricci M. Comparative study between linear and non-linear frequency-modulated pulse-compression thermography. Applied Optics. 2018;57:D32-D9.

[38] Ricci M, Callegari S, Caporale S, Monticelli M, Battaglini L, Eroli M, et al. Exploiting non-linear chirp and sparse deconvolution to enhance the performance of pulsecompression ultrasonic NDT. Ultrasonics Symposium (IUS), 2012 IEEE International: IEEE; 2012. p. 1489-92.

[39] Hutchins D, Burrascano P, Davis L, Laureti S, Ricci M. Coded waveforms for optimised air-coupled ultrasonic nondestructive evaluation. Ultrasonics. 2014;54:1745-59.

[40] Laureti S, Ricci M, Mohamed M, Senni L, Davis L, Hutchins D. Detection of rebars in concrete using advanced ultrasonic pulse compression techniques. Ultrasonics. 2018;85:31-8. 
[41] Yang R, He Y. Pulsed inductive thermal wave radar (PI-TWR) using cross correlation matched filtering in eddy current thermography. Infrared Physics \& Technology. 2015;71:469-74.

[42] Marinetti S, Grinzato E, Bison PG, Bozzi E, Chimenti M, Pieri G, et al. Statistical analysis of IR thermographic sequences by PCA. Infrared Physics \& Technology. 2004;46:85-91.

[43] Costache GN, Corcoran P, Puslecki P. Combining PCA-based datasets without retraining of the basis vector set. Pattern Recognition Letters. 2009;30:1441-7.

[44] He Y, Pan M, Luo F. Defect characterisation based on heat diffusion using induction thermography testing. Review of scientific instruments. 2012;83:104702.

[45] Yang H, Gao B, Tian G, Ren W, Woo WL. Transient-spatial pattern mining of eddy current pulsed thermography using wavelet transform. Chinese Journal of Mechanical Engineering. 2014;27:768-78.

[46] Mayr G, Stockner G, Plasser H, Hendorfer G, Burgholzer PJN, International E. Parameter estimation from pulsed thermography data using the virtual wave concept. 2018;100:1017.

[47] Bui H, Senghor F, Wasselynck G, Trichet D, Fouladgar J, Lee K, et al. Characterization of Electrical Conductivity of Anisotropic CFRP Materials by Means of Induction Thermography Technique. 2018;54:1-4.

[48] Thanh NT, Sahli H, Hao DNJIToG, Sensing R. Infrared thermography for buried landmine detection: Inverse problem setting. 2008;46:3987-4004.

[49] Zimnoch M, Oliferuk W, Maj M. Estimation of defect depth in steel plate using lock-in IR thermography. acta mechanica et automatica. 2010;4:106-9.

[50] Tian GY, Gao Y, Li K, Wang Y, Gao B, He Y. Eddy current pulsed thermography with different excitation configurations for metallic material and defect characterization. Sensors. 2016;16:843.

[51] Gao Y, Tian GY, Wang P, Wang H, Gao B, Woo WL, et al. Electromagnetic pulsed thermography for natural cracks inspection. Scientific reports. 2017;7:42073.

[52] Li Z, Meng Z. A review of the radio frequency non-destructive testing for carbon-fibre composites. Measurement Science Review. 2016;16:68-76.

[53] Arora V, Siddiqui JA, Mulaveesala R, Muniyappa A. Pulse compression approach to nonstationary infrared thermal wave imaging for nondestructive testing of carbon fiber reinforced polymers. IEEE Sensors Journal. 2015;15:663-4.

[54] Laureti S, Sfarra S, Malekmohammadi H, Burrascano P, Hutchins D, Senni L, et al. The use of pulse-compression thermography for detecting defects in paintings. NDT \& E International. 2018;98:147-54.

[55] Xu C, Zhou N, Xie J, Gong X, Chen G, Song G. Investigation on eddy current pulsed thermography to detect hidden cracks on corroded metal surface. NDT \& E International. 2016;84:27-35.

[56] Ding S, Tian G, Dobmann G, Wang P. Analysis of domain wall dynamics based on skewness of magnetic Barkhausen noise for applied stress determination. Journal of Magnetism and Magnetic Materials. 2017;421:225-9.

[57] Subhani S, Suresh B, Ghali V. Empirical mode decomposition approach for defect detection in non-stationary thermal wave imaging. NDT \& E International. 2016;81:3945.

[58] Zeng Z, Li C, Tao N, Feng L, Zhang C. Depth prediction of non-air interface defect using pulsed thermography. NDT \& E International. 2012;48:39-45.

[59] Yang R, He YJAPL. Eddy current pulsed phase thermography considering volumetric induction heating for delamination evaluation in carbon fiber reinforced polymers. 2015;106:234103. 